Florida International University FIU Digital Commons

FIU Electronic Theses and Dissertations

University Graduate School

$7-22-1998$

\title{
Circadian rhythm disruption and post-surgical recovery
}

Jeanne Anne Abdou

Florida International University

DOI: $10.25148 /$ etd.FI13101506

Follow this and additional works at: https://digitalcommons.fiu.edu/etd

Part of the Nursing Commons

\section{Recommended Citation}

Abdou, Jeanne Anne, "Circadian rhythm disruption and post-surgical recovery" (1998). FIU Electronic Theses and Dissertations. 1252. https://digitalcommons.fiu.edu/etd/1252

This work is brought to you for free and open access by the University Graduate School at FIU Digital Commons. It has been accepted for inclusion in FIU Electronic Theses and Dissertations by an authorized administrator of FIU Digital Commons. For more information, please contact dcc@fiu.edu. 


\title{
FLORIDA INTERNATIONAL UNIVERSITY
}

Miami, Florida

\section{CIRCADIAN RHYTHM DISRUPTION AND POST-SURGICAL RECOVERY}

A thesis submitted in partial satisfaction of the requirements for the degree of MASTER OF SCIENCE

\author{
IN \\ NURSING \\ by \\ Jeanne Anne Abdou
}

1998 
To: Dean DeLois P. Weekes

College of Health Sciences

This thesis, written by Jeanne Anne Abdou, and entitled Circadian Rhythm Disruption and Post-Surgical Recovery, having been approved with respect to style and intellectual content, is referred to you for judgement.

We have read this thesis and recommend it to be approved.

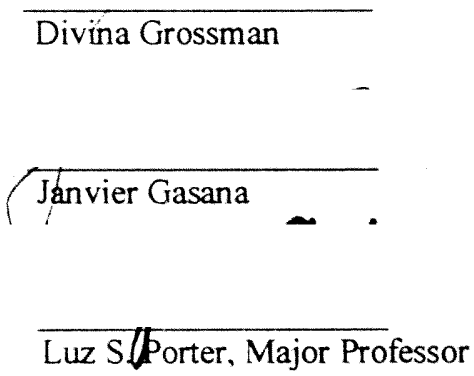

Date of Defense: July 22, 1998

The thesis of Jeanne Anne Abdou is approved

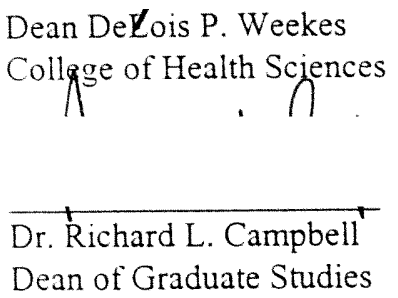

Florida International University, 1998 
I dedicate this thesis to my family for their love, patience, support and many words of encouragement throughout this journey. 


\section{ACKNOWLEDGEMENTS}

I would like to thank the following people for their help and guidance with this project: My family, for all the constant words of encouragement, love and support throughout this endeavor, I could not have done without you, Je't'aime; my friends, who helped illuminate my journey; my colleagues at Cape Coral Hospital, whose assistance and support encouraged me daily, without them, this thesis would not have been possible; and to the wonderful patients who participated in this study.

A special thank you to my committee members. Dr. Javier Gasana, for sharing his knowledge and time; Dr. Divina Grossman, for sharing her expertise on circadian rhythms and directing me to Professor Timothy Monk. Lastly, thanks to Dr Luz Porter, my major professor for her guidance, support and patience. 


\title{
ABTRACT OF THE THESIS \\ CIRCADIAN RHYTHM DISRUPTION
}

\author{
AND \\ POST SURGICAL RECOVERY \\ by \\ Jeanne Anne Abdou \\ Florida International University, 1998 \\ Miami, Florida \\ Professor Luz S. Porter, Major Professor
}

Circadian rhythms, patterns of each twenty-four hour period, are found in most bodily functions. The biological cycles of between 20 and 28 hours have a profound effect on an individual's mood, level of performance, and physical well being. Loss of synchrony of these biological rhythms occurs with hospitalization, surgery and anesthesia. The purpose of this comparative, correlational study was to determine the effects of circadian rhythm disruption in post-surgical recovery. Data were collected during the pre-operative and post-operative periods in the following indices: body temperature, blood pressure, heart rate, urine cortisol level and locomotor activity. The data were analyzed by cosinor analysis for evidence of circadian rhythmicity and disruptions throughout the six day study period which encompassed two days pre-operatively, two days post-operatively, and two days after hospital discharge. 
The sample consisted of five men and five women who served as their own pre-surgical control. The surgical procedures were varied. Findings showed evidence of circadian disruptions in all subjects post-operatively, lending support for the hypotheses. 
I. INTRODUCTION ...................................... $\quad 1$

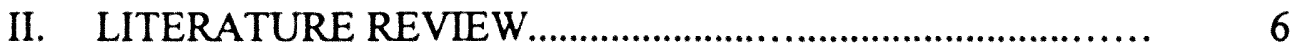

Internal Constancy, Homeostasis, and Adaptation ............ 6

Circadian Rhythms ........................................................ 8

Research Studies on Circadian Rhythms ....................... 10

Chronobiology ............................................................ 11

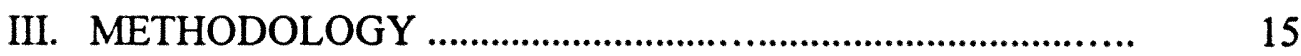

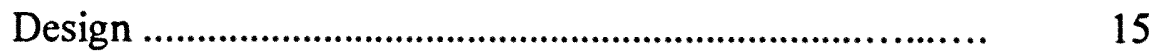

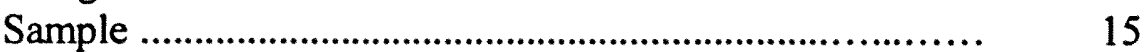

Data Collection Procedure ................................................. 16

Data Analysis ................................................................. 17

IV. PRESENTATION OF FINDINGS _.................................... 19

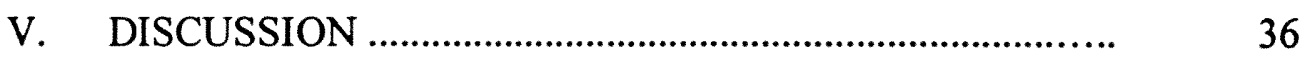

Implications for Practice .................................................. 42

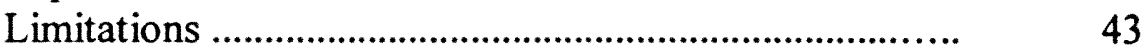

Recommendations .................................................... 44

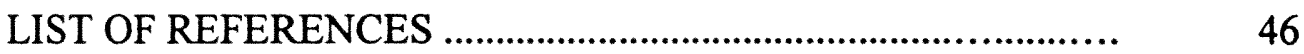

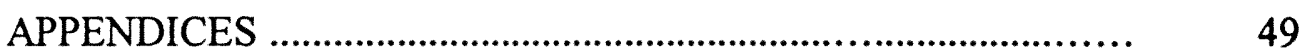




\section{LIST OF TABLES}

Page

1. Significant P-values of Systolic Blood Pressure ................................. 20

2. Significant P-values of Diastolic Blood Pressure ............................... 20

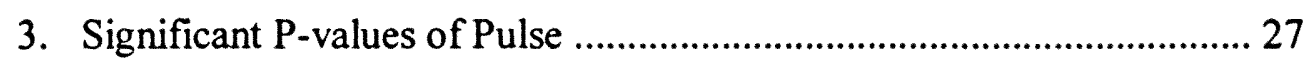

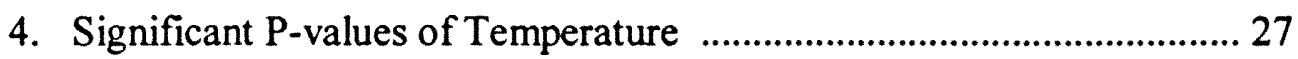

5. Comparative Percentage of Rhythmicity ........................... 35 


\section{LIST OF FIGURES}

Page

1. Bar Graph of Systolic Blood Pressure Measurements .....................22

2. Line Graphs of Systolic Blood Pressure Measurements ...................23

3. Bar Graphs of Diastolic Blood Pressure Measurements ...................24

4. Line Graphs of Diastolic Blood Pressure Measurements ................25

5. Line Graphs of Pulse Measurements ..........................................28

6. Bar Graphs of Pulse Measurements............................... 29

7. Bar Graphs of Temperature Measurements ................................... 33

8. Line Graphs of Temperature Measurements .................................34

9. Physiological Conceptual Model of Circadian Rhythmicity ......... 37 


\section{CHAPTER I}

\section{Introduction}

Circadian is defined as repeating or performing in a twenty-four hour period, especially in an organism. Circadian rhythms, patterns of each twenty-four hour period, are found in most bodily functions. The biological cycles of between 20 and 28 hours have a profound effect on an individual's mood, level of performance, and physical well being. Circadian and other biological rhythms are an integral part of the body's ability to adapt. "The ability of physiological variables to oscillate between certain limits (homeostasis) is the basic mechanism with which the body responds to challenge" (Farr, Campbell-Grossman, Mack, 1988, p 170). Optimal homeostatic function of individuals is contingent on synchrony of circadian cycles with each other and the external environment. Loss of synchrony among physiologic rhythms results in evidence of external stressors. To maintain an optimal state of health, internal rhythms are required to maintain synchronization. Studies reveal that when an organism is stressed or perceives stress, the neural, hormonal and cellular systems respond by altering their normal timing relationships (Farr et al., 1988).

Nurses assist patients in post- surgical recovery by implementing measures to prevent postoperative complications and restore optimal physiological functioning. Generalized nursing care for the postoperative recovery period revolves around the restoration of homeostasis: through the provision of nutritional support, pain control, and increasing activity levels to preoperative status. Traditionally this care has been very rigid in its delivery based on the needs of the institution rather than the individual (client). Consequently, homeostasis is disrupted coupled with dysynchrony of the client's 
circadian rhythm as demonstrated by decreased activity levels, body temperature and erratic pain control.

Further investigation of this dysynchrony will help to explain possible delay and/or complications that occur in the post surgical recovery period. Research has shown that the internal time clock is partly influenced by neural and metabolic processes. Awareness of circadian rhythms and biological time may serve as a major tool in preventive health problems.

\section{Purpose:}

The purpose of this study was to investigate the disturbance of circadian rhythms in post-surgical patients and to determine its effects on the post-surgical recovery period.

Nurses assist patients in their post-surgical recovery period. Observing and integrating the intrinsic rhythm of the body can be useful in determining an individual's health status. There are numerous body functions that follow predictable patterns. These patterns or rhythms are influenced by parts of the brain and external (environmental) factors. "Hospitalization disrupts a patient's external schedule and frequently, his biorhythmic cycles as well" (Fraser, Filler, 1989, p 32). "Rhythm studies have shown that environmental cues play an important part in maintaining normal rhythmicity" (Farr, et al, 1986). Nurses may be able to affect post-surgical recovery by initiating external factors that encourage a patient's pattern to return to normal. Alterations in circadian rhythmicity following surgery appear to be related to slower recovery (Farr, et al, 1986). Research into circadian patterns increases awareness, which can help tailor nursing care to patients' needs. Routines that compliment the intrinsic pattern of the body promote a 
feeling of emotional and physical well being. Diagnosis and treatment of some illnesses can be determined from the study of circadian rhythm or biological time. In some instances, the illness may alter the pattern of circadian rhythm, where other illnesses show exaggerated or decreased symptoms at a particular biological time.

Delays (extended hospitalization) and complications prolong the post-surgical recovery period, therefore impede the return of physiological functioning. This study helped to explain and promote stability of the patients physiological functioning in the post-surgical recovery period.

\section{Problem Statement}

- General Problem:

What are the disruptions of circadian rhythms in post-surgical patients, and what are the effects of such disruptions in their recovery?

- Specific Problems:

1) What are the effects of anesthesia/surgery on circadian rhythms in post-surgical patients?

2) What is the mean time interval needed for resynchrony of disrupted circadian rhythm in post-surgical patients?

3) What is the relationship between circadian rhythm disruption and different types of surgical procedures?

\section{- Definition of Terms:}

Circadian rhythm: a cyclic pattern repeating or performing in a twenty-four hour period. The patterns are characterized in the body by temperature regulation, heart rate, and 
blood pressure measurements. These patterns use metabolic and biological parameters such as exogenous and endogenous rhythms and emotional functioning.

Endogenous rhythm: originating within the organism

Exogenous rhythm: originating outside the organism

Post-surgical recovery: the return of homeostasis and return of pre-surgical activity and functioning in post-surgical patients

Post-surgical: in this study as after receiving general anesthesia.

Homeostasis: equilibrium of physiological functioning for an individual to maintain optimal health status.

Resynchrony: Time needed for rhythmic patterns to return to previous state after disruption. To be synchronous, occurring at the same time and rate.

\section{- Significance:}

This research study may be very useful in clinical nursing by observing and integrating the rhythms of the body can help the client's health status. "Data confirm an interrelationship between circadian rhythm and mental and physical illness" (Murray, 1985). It is important for health care providers to realize the importance of intervening with the understanding of the compensatory and adaptive processes that occur. The physiological presentation of a client's disease becomes a threat to the steady state between internal and external environments. Function becomes disordered and pathophysiology manifests in emotional, biologic and metabolic changes. Through a better understanding of clients physiological functioning and their recovery period, nurses have more insight into biological rhythm changes and adaptive processes. 
Length of stay may be decreased, which in turn is cost-effective for the institution and clients. Other advantages may be in terms of improved patient satisfaction and outcomes. New treatment approaches may arise from new knowledge obtained in this study. It is projected that the knowledge gained can help to explain phenomena that otherwise would go unanswered. Patient care can be planned with the thought in mind that homeostasis will be maintained after the body's need to rewind its circadian clock. Further studies will increase knowledge in this area. 


\section{Chapter II}

\section{Review of the Literature:}

The traditional concepts of human physiology and homeostasis reveal fluctuations in physiological and pathological processes that occur in patterns corresponding to a set calendar or clock (Cooke, 1994, p 2568). The well-orchestrated processes encounter metabolic, neural and cellular function changes. These changes may occur due to physical or mental illness, infection or medications.

\section{Internal Constancy, Homeostasis and Adaptation:}

Claude Bernard, a French physiologist in the $19^{\text {th }}$ Century, developed the biological principle that for life there must be constancy or "fixity of the internal milieu," despite changes in the external environment. Constancy refers to the physiological and biochemical static processes of homeostasis. Other physiologists emphasized the importance of involuntary neural control of the body's chemical control as responses to stimuli. The dynamic nature of the physiologic and chemical control is necessary for the concept of homeostasis and adaptation. Homeostasis refers to the body's necessary adjustments made rapidly to maintain its internal state. Adaptation refers to the responses an individual makes to function adequately under changes in the external environment or stimuli. Adaptation to voluntary or involuntary stimuli in the internal or external environment is necessary for health.

Circadian rhythms develop throughout the life cycle. Mental efficiency and performance have been related to rhythm in temperature and catecholamine excretion in the adult (Murray, 1985). Drugs can alter circadian rhythms. The shift in circadian rhythm may alter the rhythm of central nervous system function, such as barbiturates (i.e. 
sodium pentobarbital). These drugs suppress the normal rhythm of adrenal hormones, which may account for some of the hangover effect, mental blunting, and confusion that frequently accompany their use (Murray, 1985). Confusion is one of the most common experiences of an older post-surgical patient. This is aggravated by social isolation, restraints, and sensory deprivation. Nighttime confusion can be reduced by caution in the use of drugs, especially narcotic analgesics and sedatives.

"In the absence of normal social and environmental time cues, the biologic clock tends to be free-run" (Smolensky, 1996, p 12S). The body's circadian processes and functions become disrupted and desynchronized. Anesthesia medications interrupt the internal timing cues and scramble normal wake-sleep routines. During this process, functions vary from normal routines and associations. "In everyday life, the phasing of human circadian rhythms is set, or said to be synchronized primarily by sleep in darkness, activity in light" (Smolensky, 1996, p 13 S). The 24 hour period after surgery varies, but the concept of time is altered. Client's perception is different from reality, thereby causing a perceived stress. Post surgical recovery requires hemodynamic and homeostatic stability coupled with resynchrony of the circadian rhythm. Homeostasis regulates the moment-to-moment changes in the external environment. Chronobiologic mechanisms prepare the client to cope with the external environment. Adaptation occurs with integrality of the internal and external milieu and clients perception of reality. Man's biological rhythms respond to recurring cues in his environment. The external rhythms are correlated with the external fixed time structure existing in his environment to insure that vital physiologic events occur at the appropriate time (Bassler, 1976, p 575). The complex patterns of rhythmic cycles need to be integrated with environmental 
stimuli. They also need to be integrated internally. "This functional integration occurs at all levels of physiologic organization to promote smooth coordination between and among all cells, tissues, and organ systems. This matching of internal periodicities to rhythmic environmental changes is of the utmost importance in maintaining optimum daptive ability" (Bassler, 1976).

\section{Circadian Rhythms:}

Human circadian clocks are set or reset each day by environmental cues, termed, zeitgeber or synchronizers (Smolensky, 1996, p 12S). The most dominant cue is light and dark regulating the daily sleep-wake routine. Resynchronization is experienced by persons who encounter alteration in activity-sleep cycles. This temporary alteration is seen in shift-work rotation and time zone travel. In the health care setting dysynchrony occurs with administration of anesthetics, analgesics and barbiturates. "Normal" schedules of patients are altered and may be avoided during hospitalization. Circadian rhythms in critical bioprocesses give rise to prominent day-night patterns in human diseases and their symptoms (Smolensky, p $13 \mathrm{~S}$ ). The challenge in illness is to achieve and maintain homeostasis. Anticipation of the needs of the body is an integral part of reactive homeostasis. Data reveal decreased rhythmicity after surgery in animals (Farr, Campbell Grossman, Mack, 1988, p 174). Circadian rhythm disturbance alters reactive homeostatic cues. In conjunction with the circadian rhythm disturbance, synchrony is lost between an individuals internal rhythm and environmental cues following surgery. During the post-surgical period close attention to signs of circadian disturbance is necessary to determine a patient's needs. Assessment cues may include poor sleep patterns, decreased vigilance, decreased attention span, irregular body temperature 
fluctuations, and erratic pain control. Alterations in circadian rhythmicity following surgery appear to be related to slower recovery of the postoperative period (Farr, et al., p 175). Rhythms can be classified as both endogenous (internal) and exogenous (external). "Many human endogenous rhythms for example blood pressure, heart rate, body temperature, and electrolyte excretion, follow both daily and monthly cycles" (Tom and Lanuza, 1976). Corticosteriods also demonstrate a predictable daily rhythm (Tom et al., 1976). Rhythms are an integral part of life and occur to accommodate changes in life-style and life cycles. It is important to understand that physiologic variables such as body temperature, pulse rate, blood pressure, serum and urinary control, electrolytes and sensory acuity, all have been shown to have circadian rhythms. These measurements have varying mean measurements, therefore a range of values is considered "normal." Another concept to understand when discussing circadian rhythms is the patterns change according to changing environments, classifying them as highly susceptible. As discussed in the symposium on biological rhythm (Tom, et al., 1976), "just as internal body functions change throughout the day, so does the way the body responds to various stimuli. For example in studies with both animals and humans it has been shown that the same dose of the same drug given at different times of day produce different effects". The classes of drugs evoking this phenomenon include barbiturates, antihypertensives, and antihistamines.

To understand circadian rhythms, research has shown that biological rhythms can be disrupted. The disruption can be tangibly evaluated through the physiological variations seen in temperature, pulse rate, blood pressure, and excretion of urinary cortisol. 


\section{Research Studies on Circadian Rhythms:}

It is known that physical illness can disrupt normal cyclic patterns. Rhythmic cycles are prompted, or entrained, by external cues that help to maintain normal or near-normal functioning patterns (Farr, Keene, Samson, Micheal, 1984). The article by Farr further states "during hospitalization and surgery these cues are removed and replaced by novel repetitive and nonrepetitive cues imposed by the hospital environment." The body responds to these cues by loss of rhythmicity, or disruption of circadian rhythms. In a study involving eleven surgical patients, data indicate that urinary excretion of catecholamines; cortisol and electrolytes rhythms were altered (Farr, et al., p 140). Farr et al (1984) reported that circadian patterns of temperature, blood pressure, heart rate and excretion of urinary metabolites were altered following surgery in hospitalized patients.

Farr has investigated circadian rhythms in numerous research studies. An article titled Circadian Disruption and Surgical Recovery published in Nursing Research, May/June, 1988, suggests that the degree of circadian alteration, temperature and activity rhythms are altered following surgery and is positively related to the time needed for recovery and return of rhythmicity. The amount of time required for the body's rhythms to adapt to different environments and factors varies for each individual. In the article, Circadian Rhythms of Mental Efficiency and Performance, (1976). Dorothy M. Lanuza, $\mathrm{RN}, \mathrm{MSN}$, states, "desynchronization of the body's biological rhythms may cause the following symptoms: subjective fatigue; sleepiness at inappropriate times; hunger at inappropriate times or anorexia; constipation; nervousness, tension; decreased mental alertness, at least during the first two days; less efficient performance (slower reaction 
time)". To ensure optimal health and functioning it is necessary to become aware of physiological and external factors that effect homeostasis. As stated by author M. Moore-Ede (1985), "synchrony of circadian cycles with each other and the external environment is necessary for optimal homeostatic function".

\section{Chronobiology:}

Chronobiology is the branch of science that objectively explores and quantifies mechanisms of biological time structure, including rhythmic manifestation of life (Halberg, et al., 1977). This science encompasses the study of biological rhythms. As stated synchronizers influence these rhythms. Rhythms are highly susceptible to drugs. Knowledge of these rhythms can be important when designing treatment plans, protocols, and interpreting therapeutic results (Pauly, 1983).

Chronobiology uses time-dependent reference intervals to describe cyclic changes. Intrinsic time dependent changes in biological function may be cyclic, pulsatile, or random (Cooke, 1994, p 2570). Tangible information regarding these changes can be described mathematically as the frequency of changes. Acrophase is the highest point, or highest point of change. Half the distance between the highest and lowest points is defined as amplitude and mesor is the mean value of change in the cycle. Cyclic changes may account for an increase in accuracy in determining risk or perceived stress areas for patients. Therefore, it may assist in providing adaptive or preventative measures to ensure optimal health for patients.

In previous research studies on circadian disruption, data were analyzed for rhythmic changes using cosinor analysis (Farr, et al., p 171). In the cosinor analysis, data are fitted to a cosine curve. From the fitted curve, the following parameters can be 
calculated: percentage rhythmicity, mesor, acrophase, amplitude, and phase-shift (Farr, et al., p 171).

\section{- Theoretical Framework:}

The concepts of Roy's adaptation model are applicable within many practice settings in nursing. Callista Roy defines the goal of nursing as the promotion of adaptive responses in relation to the four adaptive modes: physiological, self-concept, role function and interdependence. "Adaptive responses are those that positively affect health" (Roy \& Reihl, 1980). Adaptation responses are elicited by internal or external stimuli. The adaptive system has inputs of stimuli and adaptation level, outputs as behavioral responses that serve as feedback, and control processes known as coping mechanisms (Roy \& Andrews, 1991). Outputs of a person are responses to stimuli. These responses can be both internal and external and are the person's behavior.

The regulator and cognator coping mechanisms are paramount players in the concepts of circadian rhythm and post-surgical recovery. The regulator mechanism responds automatically through neural, chemical and endocrine coping processes. The information is automatically processed and an unconscious response is produced (Roy \& Andrews, 1991). The cognator mechanism responds to inputs from internal and external stimuli. These stimuli involve psychological, social, physical and physiological factors. The responses from the regulator system are also processed.

The parasympathetic and sympathetic systems are regulators in the body during illness, wellness, and surgery. The body adapts to changes, either internal or external by precisely balancing compensatory mechanisms of heat production, heat conservation or 
precisely balancing compensatory mechanisms of heat production, heat conservation or heat loss as seen in temperature regulation. The cardiac system regulates heart rate, blood pressure, and cardiac output to maintain hemodynamic stability. The cyclic patterns of the internal body decrease or increase speed to accommodate or adapt to changes evoked by surgery, anesthesia or a changed external environment. Assessment in the post-surgical period focuses on the physiological mode described in the Roy Adaptation Model. Observation of vital signs; temperature, heart rate, and blood pressure tangibly provide an evaluation of the regulatory systems in the body. The goal of nursing outlined in Callista Roy's model is to contribute to the overall goal of health. Nursing actions enhance the interaction between the patient and the environment. Adaptation occurs during this interaction with the patient's changing environment. Health is the process of becoming integrated and adaptive to meet the external and internal stimuli. Nursing activities should promote the adaptive responses to the changing environment, thus becoming integrated and maintaining a steady state.

\section{Research Question:}

What are the effects of anesthesia/surgery on circadian rhythms in post-surgical patients?

\section{- Hypotheses:}

Based on the above, two hypotheses were formulated:

1. The circadian rhythm of surgical patients during the pre-operative period is different

from that of the pre-operative period as measured by fluctuations in:

1.1 Body temperature

1.2 Blood pressure

1.3 Heart rate 
1.4 Urine cortisol levels

1.5 Locomotor activity level

2. Surgical patients who show greater disruptions in circadian rhythm require a longer period to achieve resynchrony than those surgical patients who show less circadian rhythm disruptions.

\section{- Operational Definition:}

In this study, surgery was the independent variable, which was neither actively manipulated nor controlled. The dependant variable, circadian rhythm disruption was measured by changes in vital signs, (temperature, B/P, heart rate), locomotor activity urine cortisol levels. 


\section{Chapter III}

\section{Methodology}

Research Design:

The comparative correlational research design was used in this study to test the hypotheses. To determine circadian rhythm disruption, body temperature, heart rate, and blood pressure measurements were recorded. Post-surgical recovery was measured by locomotor activity utilizing patients' activity diary and urine cortisol level. Data were collected from the sample of ten subjects during the pre-operative and post-operative periods, while hospitalized and then 2 days after hospital discharge. Potential subjects were approached in person, on the day of their pre-operative testing appointment.

In order to evaluate a circadian rhythm disturbance, the following criteria were observed:

A.) Body temperature, heart rate and blood pressure measurements every 2 hours during the hours of 0800-2200 for 48 hours. Specifically, 2 days before surgery, post-operative day $\# 1$ and $\# 2$, and 2 consecutive days after hospital discharge.

B.) Activity level data as described in patient activity diaries and urine cortisol levels obtained four times a day.

Sample:

The sample in this study was comprised of five males and five females, age thirty-two to sixty-seven years old who served as their own pre-surgical control. The target-population for this surgery consisted of patients hospitalized for surgery. The sample was derived from surgical patients admitted in an urban hospital in southwest Florida. Sampling was done by convenience utilizing a computerized list of patients 
scheduled for pre-operative testing. This included patients from telemetry and non-telemetry units, neurology and oncology units, orthopedic and general vascular surgery units and critical care surgical units. The prospective subjects were informed about the purpose and procedure of the study. The risks and benefits were explained and they were told that they could withdraw at any time. Those who agreed to participate were requested to sign an informed consent and were assured of the confidentiality of data collected.

Instruments:

Blood pressure measurements were obtained by Datascope automatic blood pressure cuffs every two hours and recorded in the internal memory. The Datascope was used to standardize procedure and prevent interrater reliability errors.

Body temperature measurements were obtained by standard probe tympanic electronic thermometers. The thermometers were equipped with liquid crystal display (LCD) and checked for accuracy everyday using the proper methods recommended by the manufacturer. To ensure uniformity, temperatures were taken tympanically every two hours and recorded on a graph sheet. Tympanic temperatures, apical heart rate and brachial blood pressures were monitored according to standardized protocols.

Activity level were documented by participant-written activity diaries and correlated to urine cortisol levels. The urine specimens were collected and frozen for subsequent assay. The urine specimens were assayed for 17-ketosteroids using the standard procedure utilizing modified colormetric assay. Data Collection:

Data collection was started once approval from appropriate review committees 
(URC, Florida International University and agency IRB) was secured. The data were collected seven times a day at two hour intervals during normal waking hours, between the hours of 0800 and 2200 , during the two pre-operative days, the first and second days post-operatively, and two consecutive days after hospital discharge. The data collected at these specific times were temperature, apical heart rate, systolic blood pressure, and diastolic blood pressure. Urine sampling was collected and frozen four times a day for subsequent assay for cortisol. During data collection, the subjects were asked to complete a brief activity dairy to correlate with urine cortisol levels. Sampling times were chosen to approximate a typical wake/sleep cycle and minimize the effects of data collection.

The data were recorded on individualized graph sheets for each subject. The graph sheets were placed in a notebook secured in a locked file cabinet for the duration of the study. These data will be kept for three years more and then appropriately discarded.

Data Analysis:

The research data obtained were analyzed utilizing cosinor analysis: In cosinor analysis, data are fitted to a cosine curve. From the fitted curve, the following parameters can be calculated: percentage, rhythmicity, mesor, acrophase, amplitude, and phase-shift (Farr, et al., p 171). Data for all variables can be examined to determine whether they display a circadian variation. "Cosinor analysis calculates the best unbiased estimates of population parameters such as acrophase (a measure of timing)" (Farr, Keene, Samson, Michael, 1984, p 140-146). Amplitude is also measured to provide information as to the extent of rhythmic changes. The data obtained from the subjects pre-surgically and post-surgically were examined for intra-individual relationships as well as acrophase (the 
highest point of change), mesor (the mean value of the change in cycle), amplitude (half the distance between the highest and lowest points) and percentage rhythm (Farr, Keene, Samson, Michael-Jacoby, 1986, p 106). The analysis will help determine the presence of rhythmic patterns and/or variations. 


\section{CHAPTER IV}

\section{PRESENTATION OF FINDINGS}

This study was designed to show circadian rhythm disruption in post-operative patients. Further investigation was done to measure the extent of circadian disruption and its effect in post-operative recovery. The sample size in this study consisted of ten subjects. Attrition for the study was $33.3 \%$ (5). Many subjects left the study because of unexpected commitments, tedious data collection, an extended testing period, and loss of interest. Non-approval from an insurance company and cancellation of surgery prevented one subject from completing the data collection. The sample consisted of five men and five women undergoing surgical procedures. The subjects ranged in age from 32-67 years, with a mean age of 45.1 years. Ethnicity and level of education were not included in the demographics of this study. These variables do not affect circadian rhythm patterns. The ten participants served as their own pre-operative control.

In order to evaluate circadian rhythm disturbance, the following criteria were observed:

A) Body temperature, heart rate, and blood pressure measurements every 2 hours during the hours of $0800-2200$ for 48 hours. Specifically, 2 days before surgery, post-operative day $\# 1$ and $\# 2$, and 2 consecutive days after hospital discharge.

B) Activity level data, as described in patient activity diaries and urine cortisol levels obtained twice a day during two pre-operative days, two consecutive days after 


\section{SIGNIFICANT P VALUES}

\section{Table 1 \\ SYSTOLIC BLOOD PRESSURE}

Subject Preop Day 1 Preop Day 2 "Hospital Day 1 Hospital Day 2 Home Day 1 Home Day 2

\begin{tabular}{|r|r|r|r|r|r|r|}
\hline 11 & 0.060 & 0.365 & 0.105 & 0.117 & 0.681 & 0.092 \\
\hline 12 & 0.148 & 0.336 & 0.157 & 0.305 & 0.468 & 0.022 \\
\hline 14 & 0.612 & 0.103 & 0.770 & 0.122 & 0.318 & 0.659 \\
\hline 15 & 0.509 & 0.105 & 0.461 & 0.146 & 0.263 & 0.751 \\
\hline 17 & 0.198 & 0.394 & $* 0.001$ & 0.096 & 0.716 & 0.465 \\
\hline 18 & 0.375 & 0.666 & 0.320 & 0.009 & 0.153 & 0.201 \\
\hline 19 & 0.474 & 0.595 & 0.791 & 0.216 & 0.365 & 0.350 \\
\hline 21 & 0.760 & 0.254 & 0.430 & 0.783 & 0.455 & 0.041 \\
\hline 22 & 0.615 & 0.951 & 0.242 & 0.920 & 0.753 & 0.076 \\
\hline 23 & 0.345 & 0.635 & 0.433 & 0.459 & 0.064 & 0.555 \\
\hline Rhythmic (\%) & 0 & 0 & 10 & 10 & 0 & 20 \\
\hline
\end{tabular}

"significant $(p<.05)$

" highly significant $(p \leq .01)$

very highly significant ( $p \leq .001)$

"day of surgery

Table 2

DIASTOLIC BLOOD PRESSURE

Subject Preop Day 1 Preop Day 2 "Hospital Day 1 Hospital Day 2 Home Day 1 Home Day 2

\begin{tabular}{|r|r|r|r|r|r|r|}
\hline 11 & 0.306 & 0.286 & 0.787 & 0.510 & 0.886 & 0.757 \\
\hline 12 & 0.618 & 0.239 & 0.426 & 0.414 & 0.768 & 0.039 \\
\hline 14 & 0.966 & 0.265 & 0.036 & 0.784 & 0.251 & 0.135 \\
\hline 15 & 0.616 & 0.403 & 0.194 & 0.885 & 0.372 & 0.768 \\
\hline 17 & 0.693 & 0.018 & 0.764 & 0.097 & 0.971 & 0.682 \\
\hline 18 & 0.830 & 0.041 & 0.611 & 0.196 & 0.957 & 0.018 \\
\hline 19 & 0.484 & 0.220 & 0.893 & 0.569 & 0.342 & 0.151 \\
\hline 21 & 0.138 & 0.722 & 0.446 & 0.772 & 0.061 & 0.212 \\
\hline 22 & 0.058 & 0.386 & 0.327 & 0.845 & 0.501 & 0.245 \\
\hline 23 & 0.382 & 0.146 & 0.620 & 0.330 & 0.000 & 0.681 \\
\hline Rhythmic (\%) & 0 & 20 & 10 & 0 & 10 & 20 \\
\hline
\end{tabular}

-significant ( $p<.05)$

" "highly significant ( $p \leq 01)$

- very highly significant ( $p \leq .001)$

"day of surgery 
hospital discharge, and four urine samples during the first and second postoperative days.

The data, temperature, systolic blood pressure, diastolic blood pressures were analyzed using cosinor analysis. As previously described, in cosinor analysis the data were fitted to a cosine curve. From the fitted curve, the following parameters are calculated; mesor, amplitude, acrophase, and percentage of rhythmicity. Mesor is a rhythm determined average value for a variable. Amplitude is the measurement of the extent of the rhythmic change and is used to approximate a rhythm. Acrophase is defined as the peak time of the rhythmic function for a variable. The analysis provides information to determine the presence of rhythmic patterns and/or variations. The variables were compared for intra-individual relationships for each subject. Any variable with a p value of $\leq 0.05$ was statistically significant. The fitted curve for cosinor analysis was a twenty-

four (24) hour sinusoid curve. Tables 1- 4 ( p 21\& 25) depict each subject's data collected during the following phases: pre-operative, post-operative, and at home following hospital discharge. $P$ values of $\leq 0.05$ are shown to mark the statistically significant variable ( $p$ value $\leq 0.05$ appear to show rhythmicity).

\section{Research Question I}

What are the effects of anesthesia/surgery on circadian rhythms in postsurgical patients?

As depicted in Table 1, the systolic blood pressure variables showed $40 \%$ with a rhythmic pattern and $60 \%$ disrupted pattern among all 10 subjects. None of the subjects 
Figure 1

SYSTOLIC BLOOD PRESSURE

Average of All Subjects

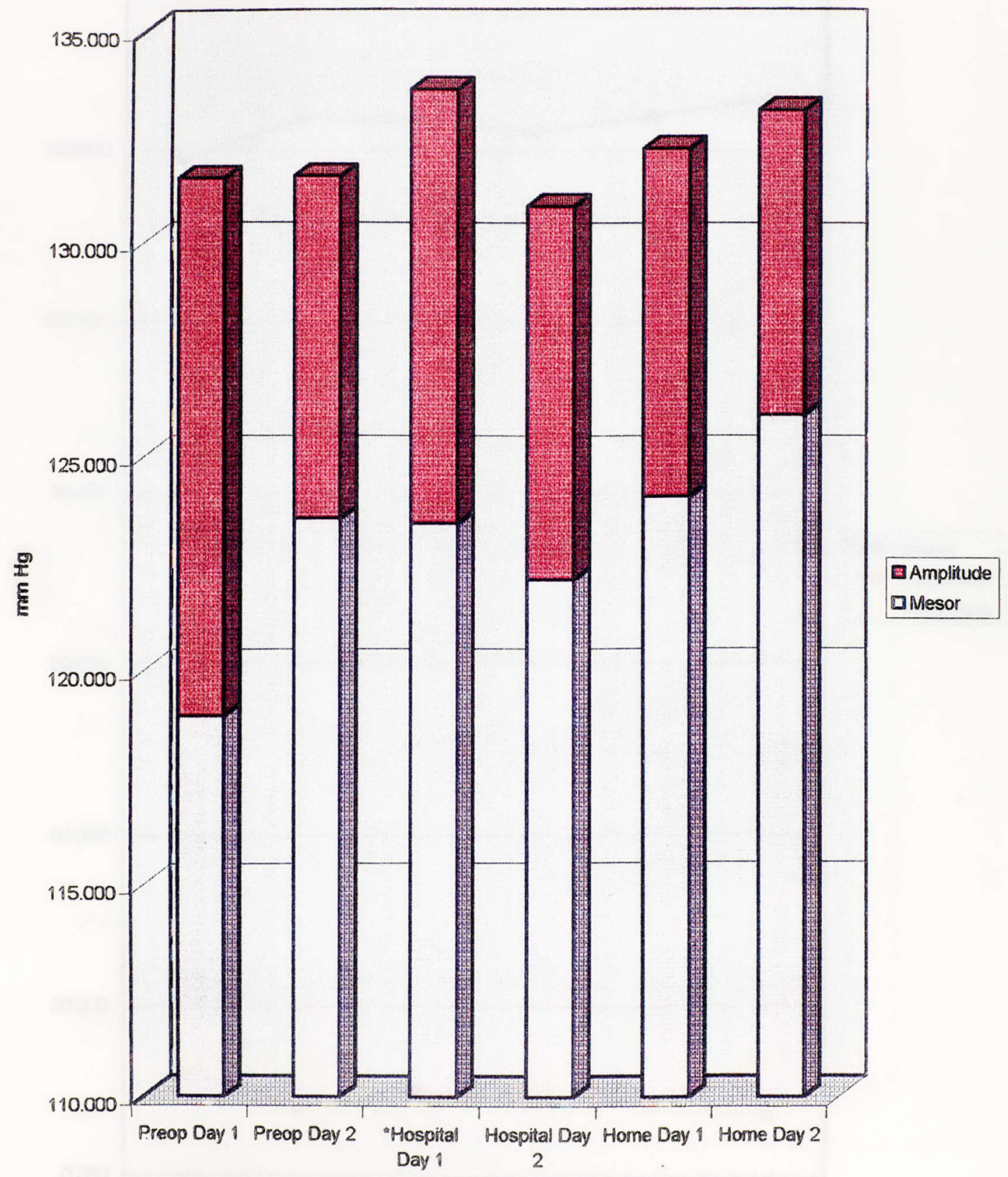


Figure 2

SYSTOLIC BLOOD PRESSURE

Average of All Subjects

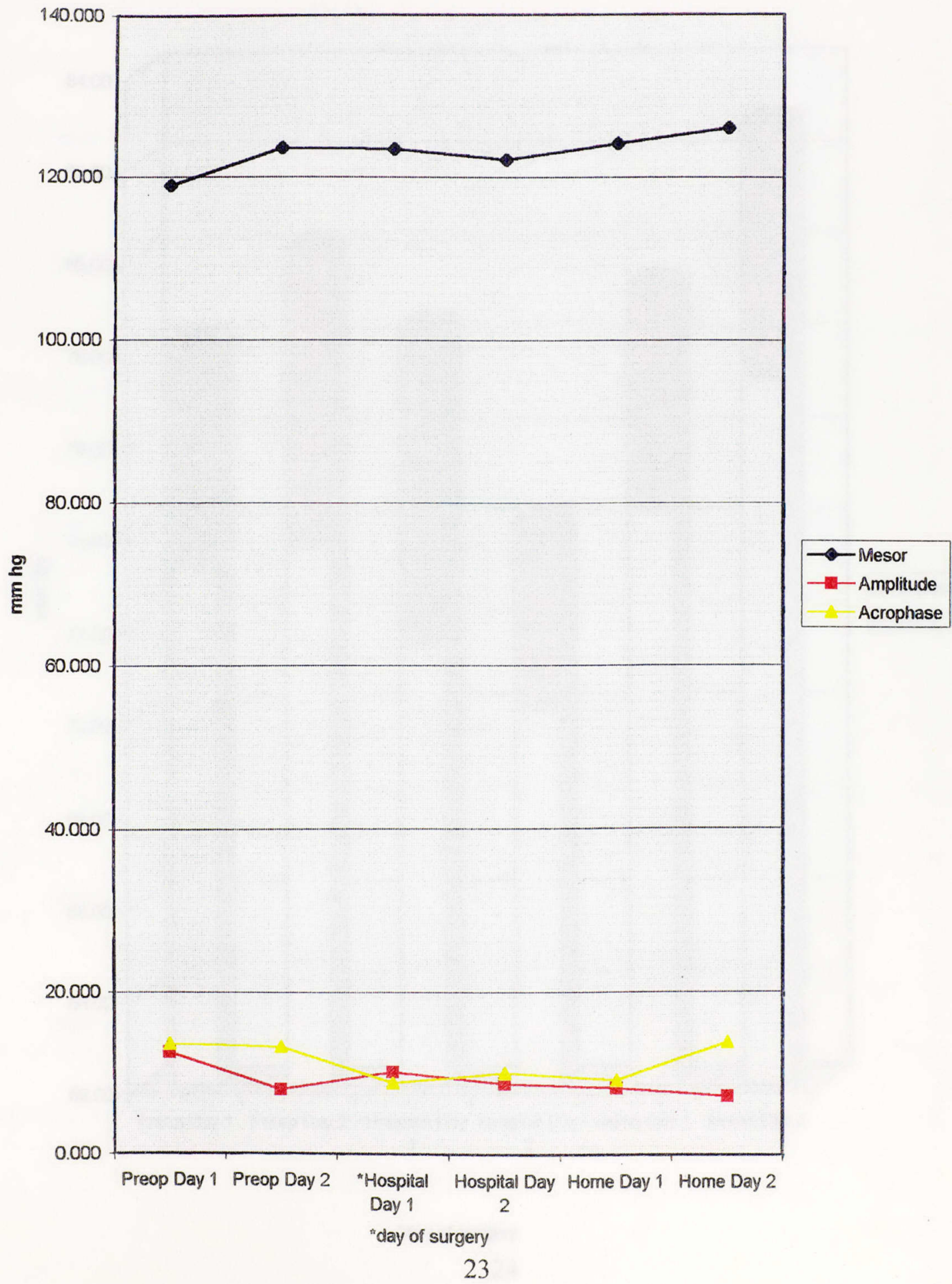


Figure 3

DIASTOLIC BLOOD PRESSURE

Average of All Subjects

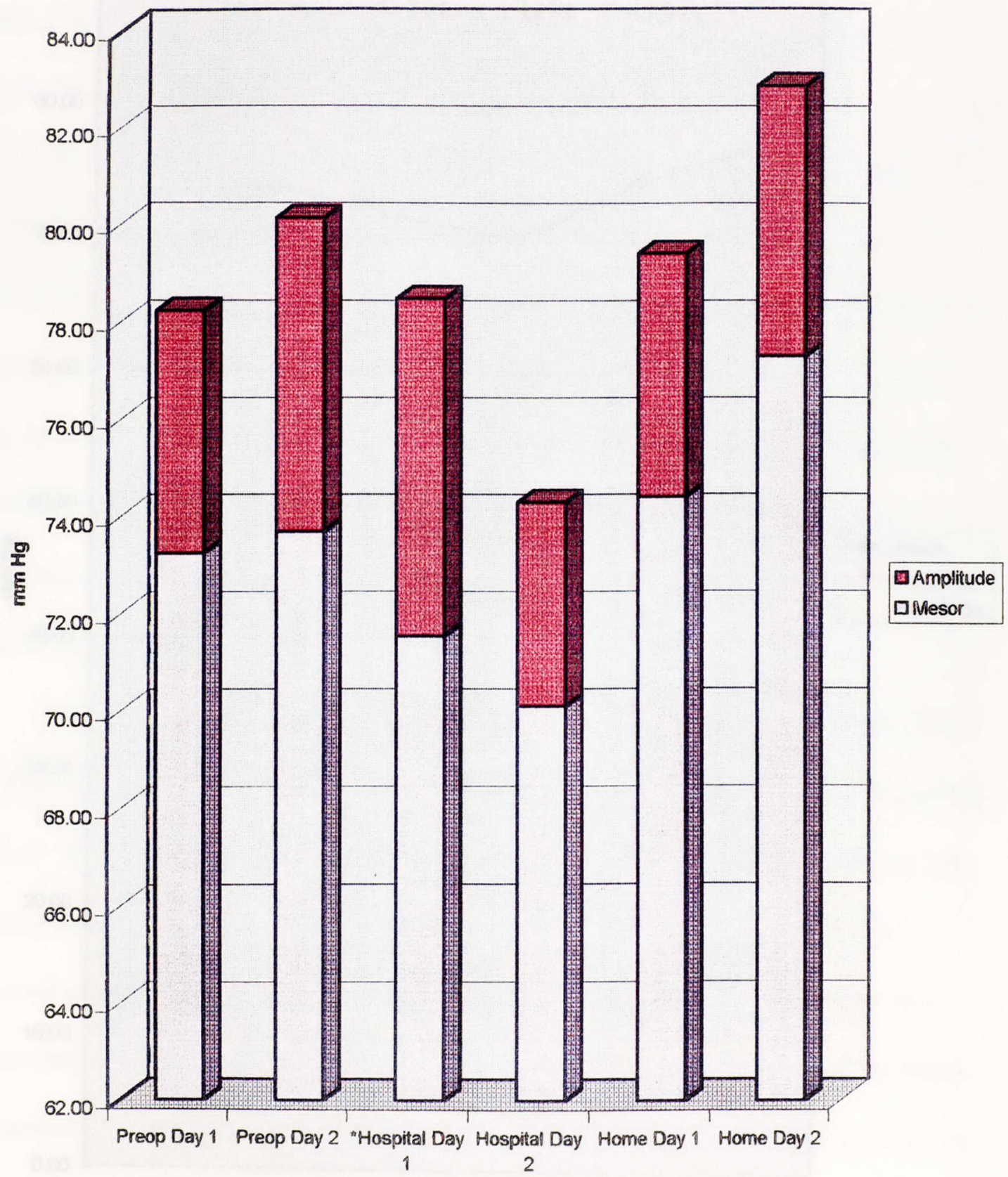


Figure 4

DIASTOLIC BLOOD PRESSURE

Average of All Subjects

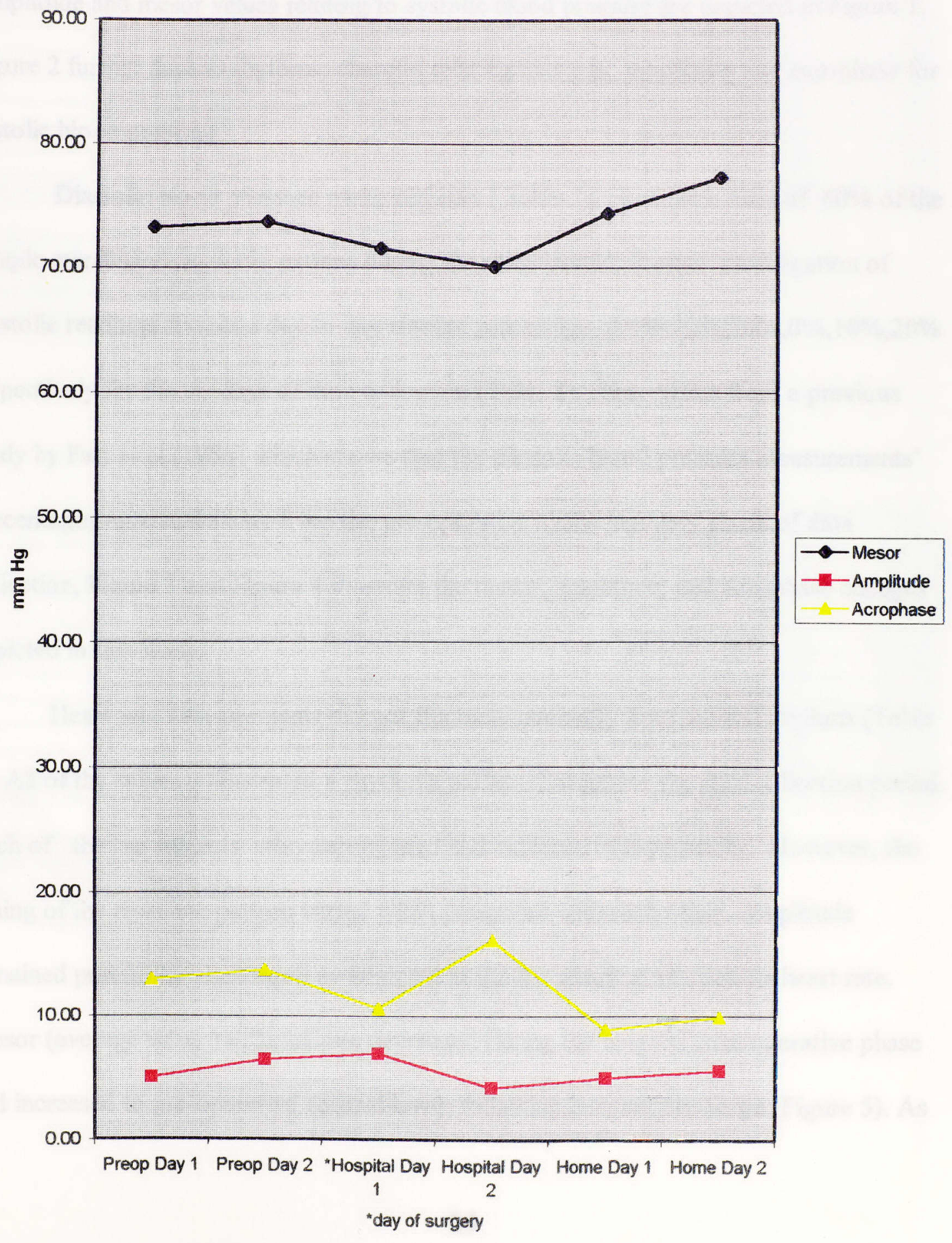


showed rhythmicity on home day \#1(24 hours after surgery), $20 \%$ of the subject's rhythms appear to return to more normal patterns on home day \#2, following hospital discharge. Amplitude and mesor values relating to systolic blood pressure are depicted in Figure 1. Figure 2 further depicts rhythmic changes relating to mesor, amplitude and acrophase for systolic blood pressure.

Diastolic blood pressure measurements ( Table 2) revealed a total of $60 \%$ of the sample manifested rhythmic pattern during the study period. Further investigation of diastolic readings reveals a day to day rhythm percentage of $0 \%, 20 \%, 10 \%, 0 \%, 10 \%, 20 \%$ respectively for the six days of data collection (Table 2). This differs from a previous study by Farr et al.(1986) which shows that the diastolic blood pressure measurements' percentages remained stable from the pre-operative to the recovery phase of data collection, Figure 3 and Figure 4 illustrate the mesor, amplitude, and acrophase changes depicted in this study.

Heart rate measurements did not fluctuate markedly from normal rhythms (Table 3). All of the subjects illustrated a rhythmic pattern throughout the data collection period. Each of the ten subjects who participated had evidence of rhythmicity. However, the timing of the rhythmic pattern varied when compared with each other. Amplitude remained practically unchanged as depicted in the bar graph of all subjects heart rate. Mesor (average value ) of heart rate decreased during the hospital post-operative phase and increased to pre-operative control levels following hospital discharge (Figure 5). As 
Table 3

PULSE

Subject Preop Day 1 Preop Day 2 "Hospital Day 1 Hospital Day 2 Home Day 1 Home Day 2

\begin{tabular}{|r|r|r|r|r|r|r|}
\hline 11 & 0.090 & 0.779 & 0.014 & 0.006 & 0.335 & 0.652 \\
\hline 12 & 0.751 & 0.009 & 0.046 & 0.589 & 0.745 & 0.685 \\
\hline 14 & 0.467 & 0.001 & 0.052 & 0.961 & 0.246 & 0.663 \\
\hline 15 & 0.055 & 0.252 & 0.211 & 0.039 & 0.598 & 0.710 \\
\hline 17 & 0.363 & 0.801 & 0.766 & 0.417 & 0.036 & 0.580 \\
\hline 18 & 0.155 & 0.765 & 0.052 & 0.196 & 0.084 & $* 0.002$ \\
\hline 19 & 0.184 & 0.033 & 0.288 & 0.349 & 0.757 & 0.067 \\
\hline 21 & 0.511 & 0.583 & 0.012 & 0.691 & 0.757 & 0.070 \\
\hline 22 & 0.885 & 0.029 & 0.562 & 0.270 & 0.036 & 0.021 \\
\hline 23 & 0.581 & 0.262 & 0.114 & 0.029 & $* 0.029$ & 0.138 \\
\hline Rhythmic (\%) & 0 & 40 & 30 & 30 & 20 \\
\hline
\end{tabular}

"significant $(p<.05)$

*highly significant $(p \leq .01)$

***ty highly significant ( $p \leq 001)$

"day of surgery

Table 4

TEMPERATURE

Subject Preop Day 1 Preop Day 2 "Hospital Day 1 Hospital Day 2 Home Day 1 Home Day 2

\begin{tabular}{|r|r|r|r|r|r|r|}
\hline 11 & 0.160 & 0.507 & 0.689 & $* 0.008$ & 0.001 & 0.301 \\
\hline 12 & 0.954 & 0.218 & 0.039 & 0.571 & 0.443 & 0.461 \\
\hline 14 & 0.108 & 0.079 & 0.802 & 0.188 & 0.392 & 0.574 \\
\hline 15 & 0.237 & 0.02 & 0.247 & 0.142 & 0.044 & 0.445 \\
\hline 17 & 0.058 & 0.094 & 0.770 & 0.202 & 0.417 & ${ }^{* * 0} 0.002$ \\
\hline 18 & 0.092 & 0.001 & $* * 0.000$ & 0.479 & 0.02 & 0.292 \\
\hline 19 & 0.840 & 0.232 & $* 0.000$ & 0.152 & 0.563 & 0.161 \\
\hline 21 & 0.122 & 0.04 & $* 0.047$ & 0.294 & 0.456 & 0.896 \\
\hline 22 & 0.041 & 0.848 & 0.278 & 0.016 & 0.443 & 0.352 \\
\hline 23 & 0.131 & & $* 0.018$ & 0.533 & 0.049 & 0.094 \\
\hline Rhythmic (\%) & 20 & 20 & 50 & 20 & 40 & 10 \\
\hline
\end{tabular}

"significant $(p<.05)$

*highly significant ( $p \leq .01)$

very highly significant $(p \leq .001)$

"day of surgery 
Figure 5

PULSE

Average of All Subjects

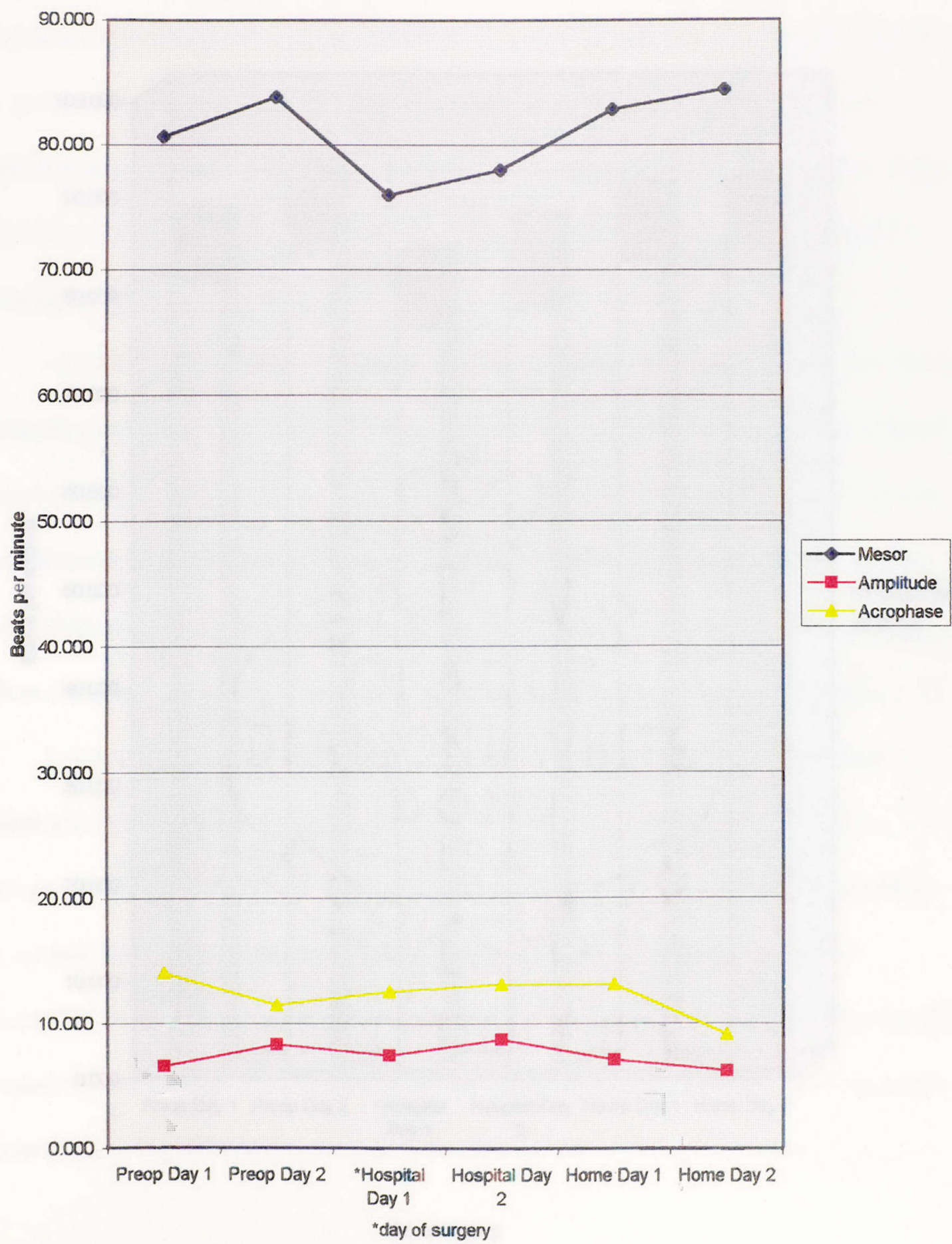


Figure 6

PULSE

Average of All Subjects

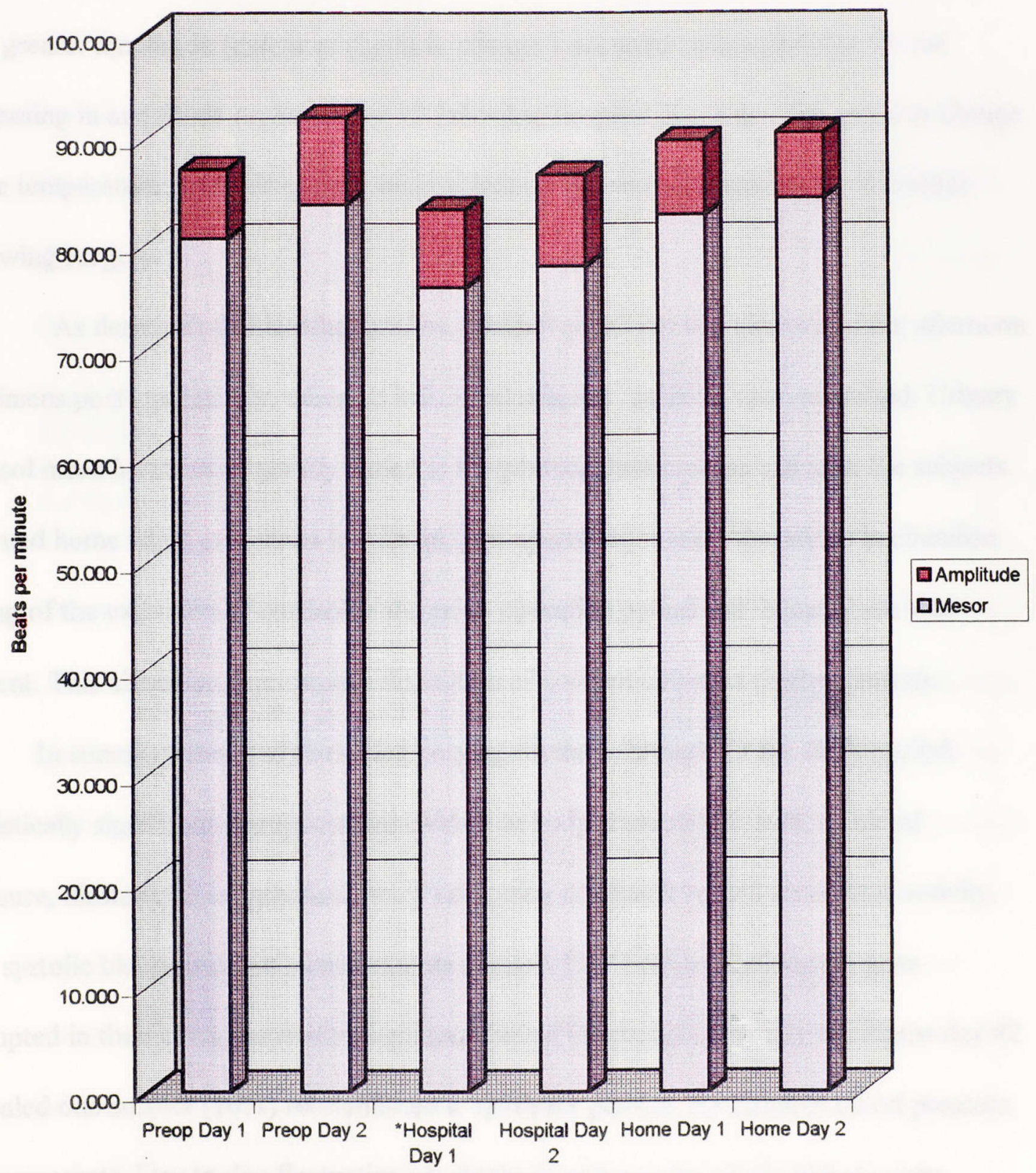

"day of surgery 
evidenced in Figure 6, circadian rhythm patterns overall remained close to normal throughout the data collection period.

As depicted in Figures 7- 8, temperature acrophase (timing of variable) was inverted on hospital day \#1 which is indicative of a shift in pattern. As depicted in Figure 7, a greater amplitude (extent of rhythmic change ) occurred on hospital day \#2 and decreasing in amplitude on home day $\# 2$ following hospital discharge. The greatest change in the temperature rhythmic pattern was evident on the second home day ( $>48$ hours) following surgery.

As demonstrated in other studies, cortisol excretion was elevated in the afternoon specimens post-operatively, when an individual subjects' patterns were examined. Urinary cortisol mean levels were greatly varied in the post-operative phase and after the subjects returned home when compared to control (pre-operative) levels. Alterations in circadian timing of the excretion of cortisol in the post- operative period and home phase was evident. This alteration represents a disruption of cortisol circadian rhythm pattern. In summary this study revealed varying rhythms throughout the study period. Statistically significant disruption was evident in body temperature, systolic blood pressure, diastolic blood pressure, heart rate, urine cortisol level and locomotor activity. The systolic blood pressure measurements showed $100 \%$ of the participants were disrupted in their circadian rhythmic pattern during the preoperative day \#1. Home day \#2 revealed one subject $(10 \%)$ reestablished a rhythmic pattern for diastolic blood pressure measurements. Day to day fluctuations in rhythmic patterns for all 10 subjects were 
evident in heart rate and body temperature during postoperative day \#2. Urine cortisol assay levels showed an increase in the afternoon level when compared to morning levels. The postoperative locomotor activity level of all subjects was decreased in fervor, however this may be viewed as subjective.

\section{Hypothesis I}

The circadian rhythm of surgical patients during the pre-operative period is different from that of the post-operative period as measured by fluctuations in:

\subsection{Body temperature}

\subsection{Blood pressure}

2.3 Heart rate

2.4 Urine cortisol levels

2.5 Locomotor activity levels

The majority of the subjects who participated in this study displayed a dysrhythmic pattern during pre-operative day \#1 for the following indices: a) body temperature, b) blood pressure, c) heart rate. The data were analyzed for evidence of rhythmic and dysrhythmic patterns. Systolic blood pressure measurements were dysrhythmic on pre-operative day \#2. The rhythmic patterns of all subjects except on temperature mesor and amplitude (Figure 8,p33) differed in the post-operative period when compared to the pre-operative (control) period. Abnormal elevations in afternoon urine cortisol levels were seen in the immediate post-operative phase and after the participants return home. The activity diaries revealed a decrease in 
activity level after return home by all participants. These findings lend support for the named indices for Hypothesis I. The findings are depicted in Tables 1-4) and Figures 1-8).

\section{Hypothesis II}

Surgical patients who show greater disruptions in circadian rhythm require a longer period to achieve resynchrony than those surgical patients who show less circadian rhythm disruptions.

As illustrated in Tables 1-4, (p 20\& 27), the percentages and statistically significant values relating to disruptions in circadian rhythm varied throughout the study period. The following variables are listed by the percentage of subjects illustrating rhythmicity on pre-operative day \#1 and compared to home day \#2, following hospital discharge. The rhythmic patterns of the subjects were seen preoperatively in body temperature. Dysrhythmic patterns are evident in blood pressure and heart rate.

These disruptions continue throughout the study period. The data, however were not further analyzed, comparing an individual's timing of circadian rhythmicity to observe the time necessary for resynchrony. The data collected in this study did not allow for testing this hypothesis because the subject's baseline circadian rhythms could not be determined from the pre-operative data. As depicted in Table 5, disrhythmic patterns were evident pre-operatively in systolic, diastolic blood 
Figure 7

TEMPERATURE

Average of All Subjects

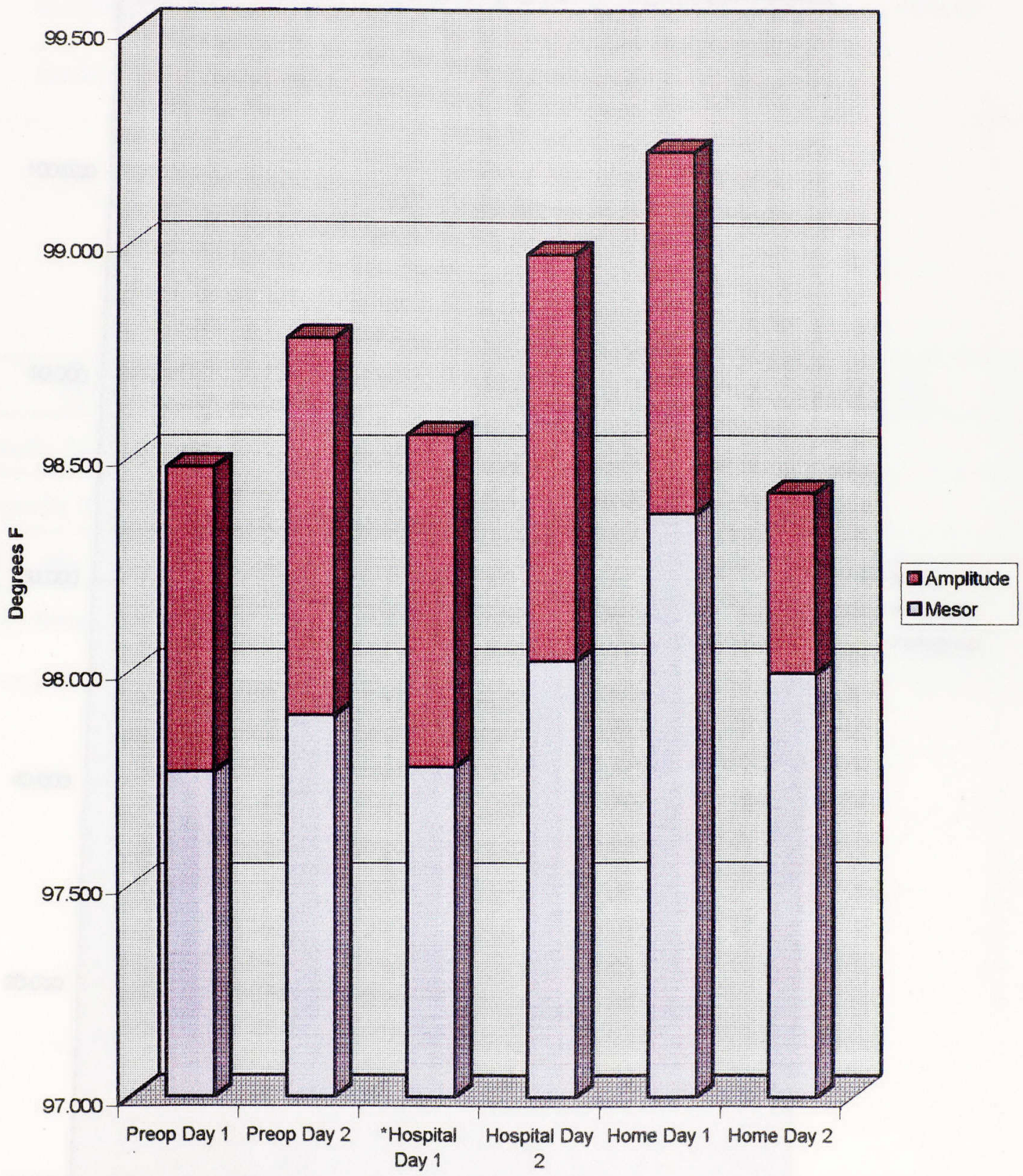

"day of surgery 
Figure 8

TEMPERATURE

Average of All Subjects

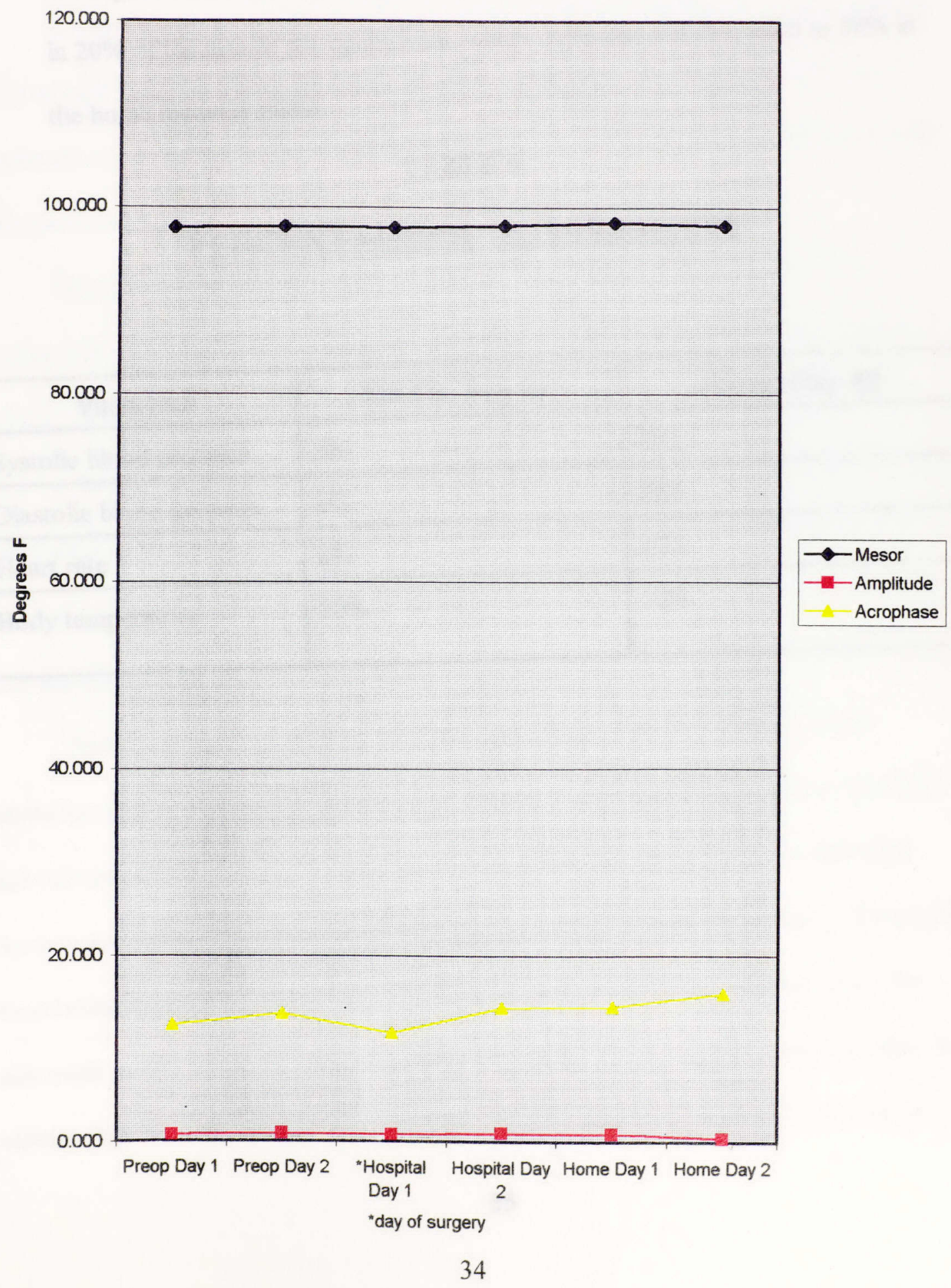


pressure measurements, and heart rate. The disrhythmic patterns continued to the home phase in $80 \%$ of the sample. Body temperature rhythmic patterns were seen in $20 \%$ of the sample pre-operatively, which continued and decreased to $10 \%$ in the home recovery phase.

\section{TABLE 5}

\section{PERCENTAGE OF RHYTHMICITY}

\begin{tabular}{|l|l|l|}
\hline \multicolumn{1}{|c|}{ Varlables } & \multicolumn{1}{|c|}{ Pre-Op Day \#1 } & \multicolumn{1}{c|}{ Home Day \#2 } \\
\hline Systolic blood pressure & $0 \%$ & $20 \%$ \\
\hline Diastolic blood pressure & $0 \%$ & $20 \%$ \\
\hline Heart rate & $0 \%$ & $20 \%$ \\
\hline Body temperature & $20 \%$ & $10 \%$ \\
\hline
\end{tabular}




\section{CHAPTER V}

\section{DISCUSSION}

In order to better spend health care dollars and improve patient care outcomes the ability of care givers to assist patients through the healing process more expeditiously is paramount as we approach the new millennium. It is for these reasons that I initiated this study on circadian rhythm disruption and post-operative recovery.

Ten subjects participated in the study that encompassed a total of six days, starting with two pre-operative days, two days of the immediate post-operative period, and two days after hospital discharge. Circadian rhythm disturbance was evaluated by observation of body temperature, heart rate, blood pressure and urine cortisol levels. Less disruption of the circadian rhythm assists clients and the health care delivery system to likely provide optimal strategies to obtain and maintain homeostasis which may decrease cost and improve patient care outcomes.

Previous research involving circadian rhythms provides information which attributes surgery to circadian rhythm dysynchrony. According to Farr, Keene, Samson, and Micheal-Jacoby (1985), “during the period of time that the individual's circadian rhythms are out of phase with each other and the external environment, the physiological functioning is sub-optimal and may add an additional burden to recovery ( $p$ 105). The schematic model in Figure 10 (p 37), from Farr et al,(1984), helps to conceptualize the relationship between normal circadian rhythms of catecholamines and 17-ketosteriods 
Figure 10

\section{A Physiological Conceptual Model Depicting the Relationship Between Altered Circadian Function Resulting From Surgical Trauma, and the Return of Normal Circadian Rhythmicity}

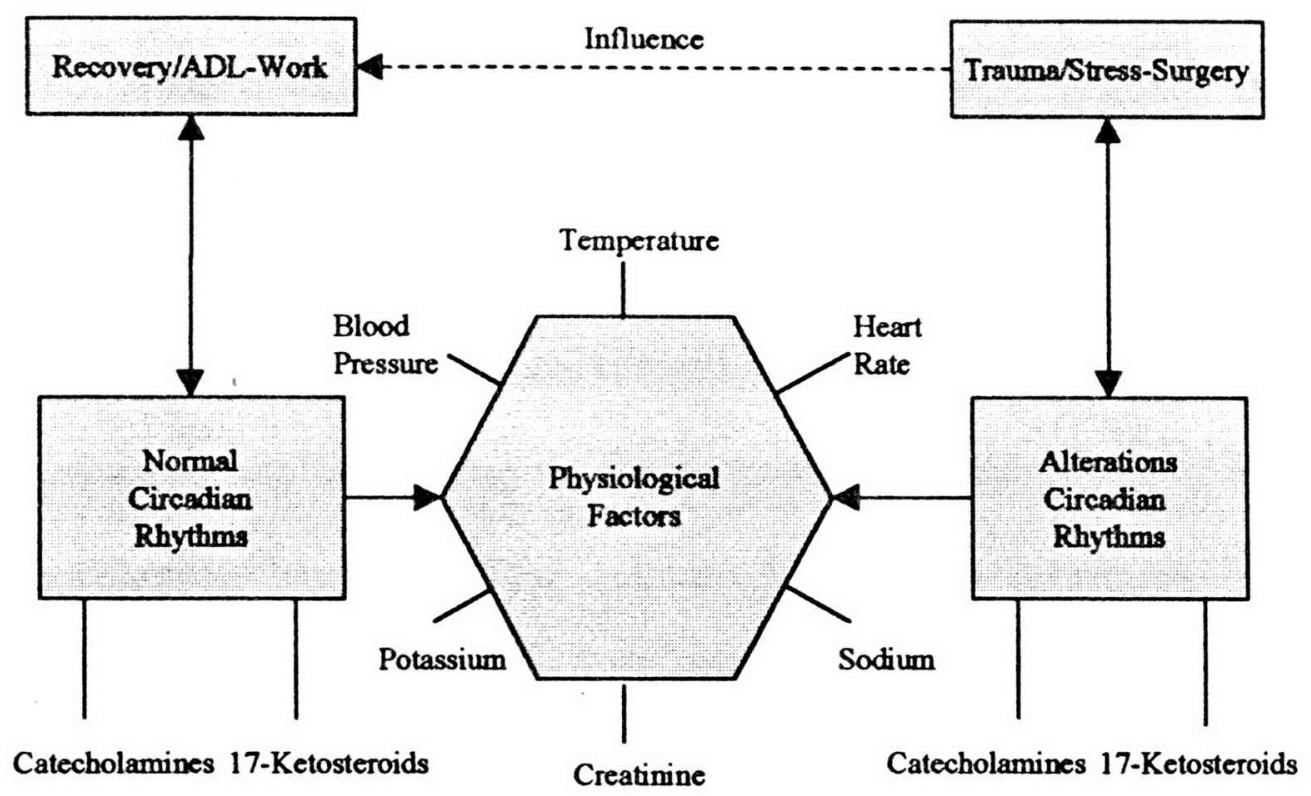


(cortisol) and the ability to perform activities of daily living or work and to show a similar association of altered circadian rhythms affected by stress, trauma, and surgery.

The literature suggests that stress, tension, and loss of synchronizing cues (daylight, noise and activity), from the external environment affect rhythmicity. "If these effects are expressed as timing alterations, uncoupling between internal rhythms and from external cycles, it may be assumed that a period of adjustment is created. During this period the subject's physiological functioning may be sub-optimal with the recovery affected" (Farr, Keene, Samson, Michael, 1984).

Disruption in rhythmic patterns of the variables were evident by the results. The assumption is made in utilizing cosinor analysis that the internal timing is based on a 24 hour clock. However, an individual's circadian rhythms may be based on varying internal timing devices. Temperature increases normally occur during the post-operative phase, which is associated with healing and the body's natural defenses to repair itself. "Because temperature rhythms reflect metabolic cycles, it may be assumed that alterations in these cycles affect the availability of metabolic substrates necessary for tissue repair"(Farr, et al.,1988). Temperature mean percentages were low. This may be explained by a comparatively short time to show disruption of a highly stable rhythm. Zeitgebers, human circadian timing synchronizers, were not determined prior to actual data collection. A trial period of data collection may have been necessary to obtain an individual's rhythmic pattern pre-operatively. During this period zeitgebers can be defined, 
allowing intrinsic rhythmic patterns to adjust to the changing environment (data collection).

Cortisol is the most naturally occurring glucocorticoid. It is necessary for the maintenance of life and for protection from stress. ACTH is the main regulator of cortisol secretion. Diurnal rhythms affect ACTH and cortisol levels. In persons with a regular sleep-wake patterns, cortisol peaks three to five hours after sleep begins. Excretion of cortisol therefore peaks in early morning upon awakening and steadily decline throughout the day. Stress has been shown to increase ACTH secretion, leading to an increased cortisol level. In addition, researchers must be aware of other factors that influence cortisol levels, such as improper urine sample procurement and errors in laboratory measurements.

The subjects in this study ranged in age from 32-67 years. This would account for differences in circadian rhythm disruption from the norm. Younger, less compromised patients have the physical ability to heal more quickly than older patients whose organ functions are normally starting to slow, therefore hampering or slowing the healing process with the added burden of circadian rhythm disturbance. The disrupted preoperative patterns can be explained by an increased stress level, interrupted work schedules for pre-operative tests, and planning for post-operative convalescence. The data suggest that surgery and hospitalization do induce changes in circadian rhythms of individual subjects. The rhythms attempt to return to a more rhythmic pattern after surgery during the hospitalization period and the home phase after hospital discharge. The 
amplitude of a rhythm steadily increases until the rhythmic pattern is obtained (MooreEde, 1982).

The primary goal of the Roy Adaptation Model is to achieve health, wellness, and stability through adaptation in a changing environment (Roy \& Reihl,1980). The balance between the dynamic environment of post-operative recovery and homeostasis can be facilitated by health care personnel. Nursing actions enhance the interaction between the patient and the environment. The untemporal environment of hospitals evince sleep disturbance and stress with its concomitant physiological responses. During the adaptation process, the nurse can provide care with insight and understanding of circadian rhythm disruption and assist the patient to the goal of health. A high degree of temporal order is paramount to characterize a healthy organism. "Hospitals are not the most normal of temporal environments with noise, light, and activity maintained throughout day and night"(Moore-Ede 1985). Research suggests health professionals should individualize patient care to promote rhythmicity. An article by Felton (1987), cites a discrepancy between the activity schedule of the individual and the environmental (hospital) schedule can result in physiological and psychological distress with decreased, less efficient performance. Rhythms affect moods, mental, and physical performance. A conscious effort should be made when making decisions regarding patient care. "In regard to patients, our awareness of their rhythms should play an important role in planning with them the best time for scheduling patient teaching sessions, rehabilitation exercises, rest periods, and other nursing procedures as well as activities of daily living" (Lanuza,1976). 
Hospital schedules conflict with patients normal schedules and patterns. Superimposed time schedules are invasive and interfere with patients' external timing. Procedures such as diagnostic tests, surgery, specimen procurement, and physical therapy require thought to attempt to approximate normal sleep-wake patterns. Individualizing care allows for less circadian disruption and increased effectiveness. Disorganization of normal circadian rhythms can be manifested by anxiety, irritability, and decreased concentration or alertness. There may also be an increase in somatic complaints such as gastrointestinal disturbances and in accident proneness (Hoskins, 1981).

It can not be disputed, that an assessment of a patient's behavior, physical assessment and interpersonal relationships are an integral part of professional nursing practice. "Following surgery, the body must respond to challenges of fluid loss, temperature alteration, and metabolic requirements which may strain or exceed the limits normally maintained by homeostasis" (Farr, et al.,1988). The body's ability to react to these postoperative challenges are also compromised with the additional alteration of circadian rhythm disruption during post surgical recovery. The combined challenges invoke inefficiencies illustrated by distuption of sleep, gastrointestinal complaints, decreased vigilance, decreased attention span, and a general feeling of malaise. It has been pointed out that alterations of circadian rhythms can lead to slower recovery, extended hospitalization, and increased health care costs. Reduction of circadian rhythm disruption should be considered as a means of improving a patient's feeling of well-being and 
reducing post-operative complications toward the goal of enhancing patient care outcomes.

Based on the study findings, it is reasonable to conclude that patients who undergo surgery experience disruption of circadian rhythms. The disruptions are evident in changes in rhythmic patterns of physiologic and psychologic functioning.

\section{Implications For Practice}

Health care has attained extraordinary technological achievements. However, rhythms of physiologic and psychological functioning are consistently variable and complex to understand. As more research data become known regarding these rhythms, health care can be planned accordingly. The study findings have several implications and potential importance for nursing research and nursing practice.

Hospital routines are day-oriented and may not coincide with an individual's normal routine. Patients are subjected to hospital routines which may conflict with their circadian rhythmic pattern and timing cues. Activities of daily living such as, bathing, ambulation, and meal times should be planned with the individual patient in mind. Less disruption of wake/sleep cycles and activities of daily living may minimize the effects of circadian rhythm dysynchrony. An awareness and assessment of the signs and symptoms of disruption is paramount in order to individualize patient care and to anticipate problems arising from circadian disruption. Disruptions are evident in altered sleep patterns, a 
decrease in attention span and vigilance, increased somatic complaints, and a general feeling of malaise.

The scheduling of medications, diagnostic tests, therapies, and surgery should be planned with consideration of the patient's wake/sleep pattern. Whenever possible, the care provided should allow reentrainment of circadian rhythmicity by providing time and place orientation, scheduling activity and rest periods, and meal times that follow the patient's wake/sleep patterns. This may include simple interventions such as opening and closing window blinds, providing clocks and calenders, and minimizing interruptions of rest periods. Nurses implementing measures to prevent post-operative complications and measures that reduce disruptions of circadian rhythmicity restore optimal physiological functioning. Awareness of circadian rhythms and biological time can serve as a major tool in prevention of post-operative complications and promote reestablishment of homeostasis.

\section{Limitations}

Limitations encountered in this study are primarily related to the research design. First, the sample size of ten was done by convenience sampling. This limits the generalizabliity of the study findings. Secondly, the data collection period encompassed six days for each subject. The protocol for data collection proved to be too complex for one data collector in a limited time frame. Thirdly, contaminating variances hampered data collection. These variances were discarded urine samples and incomplete vital sign 
measurements. Attempts to collect complete pre-operative and post-operative data were unsuccessful.

The variety of surgical procedures might have introduced a bias in the study findings. Presumably, clients, who had elective surgery as compared to clients who had surgery to prevent tertiary complications have more control over the situation. As the literature documents, increased stress increases cortisol levels and vital sign measurements. An example from this study is a 42 year old female who had a breast augmentation for aesthetic reasons versus a 67 year old male with a history of syncopal episodes due to carotid artery stenosis requiring a carotid artery endarterctomy. An increase in the excretion of urine cortisol is associated with sleep deprivation and increasing vital sign measurements

Lastly, factors such as medications, dietary intake, and varied activity schedules were impossible to control. The direction of the study findings could have been otherwise.

\section{Recommendations for Further Research}

Based on the limitations of this study, the following recommendations are hereby presented:

1) Increase the sample size and use random sampling to enhance generalizability of study findings.

2) Utilize several data collectors to ensure accurate and sufficient volume of preoperative and post-operative data. 
3) Account for the medications and pain control measures during the postoperative recovery period to evaluate their effect in circadian rhythm disturbance. 4) Design a study testing the effects of different types of surgical procedures on circadian rhythm disruptions.

5) Control threats to internal and external validity. 


\section{References}

Aschoff, J. (1965). Circadian rhythms in man. Science, 148 p 1427-1432.

Bassler, S. (1976). The origins of development of biological rhythms. Nursing Clinics of North America, 11 (4) p 575-583.

Cooke, H. and Lynch, A. (1994). Biorhythms and chronotherapy in cardiovascular disease. American Journal of Hospital Pharmacists, (51) p 2568-2580.

Davis, C., Lentz, M. (1989). Circadian rhythms: Charting oral temperatures to spot abnormalities. Journal of Gerontological Nursing 15 (4) p 34-39.

Farr, L., Campbell-Grossman, C., Mack, J. (1988). Circadian disruption and surgical recovery. Nursing Research37 (3).

Farr, L., Keene, A., Samson, D., Michael, A. (1984). Alterations in circadian excretion of urinary variables and physiological indicators of stress following surgery. Nursing Research, 33 (3) p 140-146.

Farr, L., Keene, A., Samson, D., Michael-Jacoby, A. (1986). Relationship between Disruption of rhythmicity and reentrainment in surgical patients. Chronobiologia, $13(105)$ p 105-113.

Fraser, C., Filler, M. (1989). The assessment factor most nurses forget. Registered Nurse p 32-34

Johnson, C., Hastings,J. (1986). The elusive mechanism of the circadian clock. American Scientist, 74 p 29-36

Marques, M., Waterhouse, J. (1994). Masking and the evolution of circadian rhythmicity. Chronobiology International, 11 (3) p 146-155. 
Mason, D. (1988). Circadian rhythms of body temperature and activation and the well-being of older women. Nursing Research, 37 (5) p 276-280.

Mason, D., Redeker, N. (1993). Measurement of activity. Nursing Research, 42 (2) p 87-92.

Minors, D.S., Waterhouse, J.M. (1986). Circadian rhythms and their mechanisms. Experientia 42 (1) p 1-13.

Moore-Ede, M., Czeisler,C., Richardson, G. (1983). Circadian timekeeping in health and disease. The New England Journal of Medicine, 309 (8) p 469-476.

Moore-Ede, M., Sulzman, F., and Fuller, C. (1982). The clocks that time us. Harvard University Press, Cambridge, MA.

Murray, R. and Zenter, J. (1985). Nursing concepts for health promotion. Prentice-Hall, Inc., Englewood Cliffs, NJ.

Pauley, J.E. (1983). Chronobiology: Anatomy in Time. The American Journal of Anatomy, 168 p 365-388.

Rietveld, W., Minors, D., Waterhouse, J.(1983). Circadian rhythms and masking: an overview. Chronbiology International, 10 (4) p 306-312.

Roy C. and Reihl, J. P. (1980). Conceptual nursing for nursing practice. Appleton \& Lange, Norwalk, CT.

Smolensky, M. (1996). Chronobiology and chronotherapeutics: applications to cardiovascular medicine American Journal of Hypertension,(9) p 11S-21S.

Straka, R. and Benson, S. (1996). Chronopharmacologic considerations when treating the patient with hypertension. Journal of Clinical Pharmacists (30) p 771-782. 
Thomas, K. (1990). Time-series analysis-spectral analysis and the search for cycles.

Western Journal of Nursing Research, 12 (4) p 558-562

Tom, C., Lanuza, D. (1976). Symposium on biological rhythms. Nursing Clinics of North America, 11 (4) p 572-628.

Weinert,D.,Eimert,H.,Erkert,H.,Schneyer,U.(1984).Resynchronization of the circadian corticosterone rhythm after a light/dark shift in juvenile and adult mice.

Chronobiology International, 11 (4) p 222-231. 


\section{APPENDICES}

\section{Page}

A. Statistics of Systolic Blood Pressure ............................ 50

B. Statistics of Diastolic Blood Pressure .............................. 51

C. Statistics of Pulse Measurements .................................. 52

D. Statistics of Temperature Measurements ............................. 53

E. Mean Cortisol Levels ........................................... 54

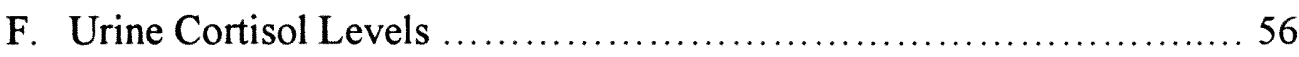

G. Research Protocol .............................................6 60

H. Subject Recruitment Letter ....................................69

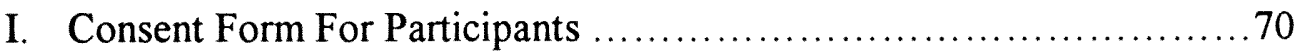

J. Consent to Conduct Research at Cape Coral Hospital .................71

K. Acceptance Letter from Vice President of Patient Services

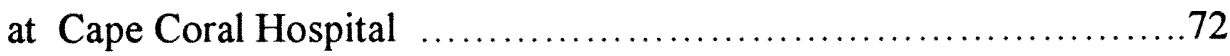


Systolic Blood Pressure

Mesor

Preop Day 1

Preop Day 2

In Hospital Day 1

In Hospital Day 2

Postop Day 1

Postop Day 2

Amplitude

Preop Day 1

Preop Day 2

In Hospltal Day 1

In Hospltal Day 2

Postop Day 1

Postop Day 2

un

Acrophase

Preop Day 1

Preop Day 2

In Hospltal Day 1

In Hospltal Day 2

Postop Day 1

Postop Day 2

\section{Preop Day 1}

Preop Day 2

In Hospltal Day 1

In Hospltal Day 2

Postop Day 1

Postop Day 2
Patlent 11 Patlent 12

$\begin{array}{ll}102.775 & 129.130 \\ 132.014 & 134.878 \\ 153.454 & 134.637 \\ 155.908 & 127.571 \\ 135.195 & 127.631 \\ 147.739 & 133.272\end{array}$

105.666

104.363

100.328

90.499

115.312

103.796
Patlent 18 Patlent 14 Patlent 17 Patlent 21 Patlent 19 Patlent 22 Patlent 23

$126.553 \quad 132.271-105.108$

$128.641 \quad 134.200$

$\begin{array}{lll}123.249 & 134.324 & 108.356\end{array}$

$\begin{array}{lll}134.289 & 134.484 & 103.837\end{array}$

$\begin{array}{lll}126.213 & 136.331 & 107.232\end{array}$
128.118 139.576 116.237 126.652

131.707

137.380
145.542

141.067

127.962

124.082

126.951
143.153
$108.718 \quad 119.573$

$101.149 \quad 118.554$

$103.694 \quad 134.693$

$97.077 \quad 133.421$

$104.221 \quad 127.047$

$107.998 \quad 116.691$

$\begin{array}{rrrrrrrrrr}61.003 & 10.724 & 3.717 & 5.755 & 4.473 & 10.939 & 2.796 & 7.108 & 3.670 & 15.589 \\ 10.478 & 2.852 & 5.302 & 2.160 & 14.802 & 8.910 & 14.388 & 5.149 & 0.989 & 15.077 \\ 16.691 & 12.507 & 12.740 & 3.561 & 3.720 & 10.396 & 16.047 & 4.187 & 16.027 & 5.868 \\ 13.348 & 8.059 & 4.369 & 16.427 & 7.405 & 13.077 & 7.775 & 8.825 & 2.947 & 5.396 \\ 10.450 & 5.239 & 18.524 & 9.575 & 4.800 & 4.439 & 4.660 & 9.445 & 5.320 & 9.201 \\ 13.778 & 8.865 & 6.599 & 3.700 & 3.331 & 6.622 & 7.488 & 10.858 & 6.146 & 4.108\end{array}$

$\begin{array}{rrr}12.927 & 22.853 & 20.485 \\ 17.985 & 22.627 & 20.764 \\ 1.920 & 18.328 & 2.980 \\ 3.959 & 4.337 & 16.812 \\ 15.663 & 5.259 & 2.196 \\ 8.172 & 24.387 & 24.090\end{array}$

$\begin{array}{rr}6.184 & 4.125 \\ 12.363 & 18.021 \\ 15.327 & 7.629 \\ 5.987 & 1.384 \\ 4.259 & 5.449 \\ 6.434 & 7.725\end{array}$

14.020

19.289

3.742

17.737

14.571

1.287

4.145

4.135

10.013

11.204

13.778

5.009

14.100

16.044

15.512

20.169

13.462

16.774

3.227

15.782

3.582

3.945

23.411

23.558

MEAN

Mesor Ampiltude Acrophase

$\begin{array}{lll}118.937 & 12.577 & 13.593\end{array}$

$\begin{array}{lll}123.553 & 8.011 & 13.218\end{array}$

$\begin{array}{lll}123.438 & 10.174 & 8.782\end{array}$

$\begin{array}{lll}122.114 & 8.763 & 10.060\end{array}$

$\begin{array}{lll}124.067 & 8.165 & 9.153\end{array}$

$\begin{array}{llr}125.981 & 7.149 & 13.809\end{array}$ 


\section{Diastollc Blood Pressure}

Mesor

Preop Day 1

Preop Day 2

In Hospltal Day 1

In Hospltal Day 2

Postop Day 1

Postop Day 2
Pallent 11 Patlent 12 Patlent 15

75.142

76.803

59.522

68.321

75.037

79.209
79.87

81.585

79.336

79.338

79.447

76.701

$\begin{array}{rr}63.92 & 82.439 \\ 61.784 & 78.706 \\ 62.381 & 80.813 \\ 49.959 & 66.533 \\ 67.982 & 82.668 \\ 63.366 & 77.765\end{array}$

77.302

78.215

79.005

82.412

83.883

82.333
56.867

55.518

58.891

58.225

58.779

67.617
77.659

80.6

73.115

78.049

87.478

85.7
87.201

80.701

73.946

75.884

68.042

81.846
64.424 68.802

68.874

65.072

69.316

75.392
Amplltude

Preop Day 1

Preop Day 2

In Hospltal Day 1

In Hospltal Day 2

Postop Day 1

Postop Day 2

Acrophase

Preop Day 1

Preop Day 2

In Hospltal Day 1

In Hospltal Day 2

Postop Day 1

Postop Day 2

$\begin{array}{rr}7.934 & 4.835 \\ 7.408 & 4.213 \\ 3.202 & 8.874 \\ 3.894 & 7.266 \\ 1.686 & 3.144 \\ 3.852 & 10.862\end{array}$

$\begin{array}{rr}4.009 & 1.376 \\ 1.94 & 4.129 \\ 18.354 & 2.092 \\ 0.949 & 3.748 \\ 6.646 & 1.002 \\ 2.906 & 7.305\end{array}$

0.756

9.559

6.196

2.08

4.825

2.202

5.286
15.74
5.106
5.445
1.59
4.35

3.67
1.223
11.013
5.799
6.581
3.667

4.354

6.154

3.108

4.584

11.321

9.022
10.46
2.877

9.71

2.322

3.866

6.427

67.602

74.164

79.404

77.109

71.292

72.846

2.257
14.008
17.555
6.641
2.703
6.836

10.627
17.098
12.846
16.035
2.612
14.655

17.488
20.207
15.607
23.467
18.011
7.452

24.787
1.518

21.957

10.962

1.017

3.867

4.54

14.646

14.811

20.744

16.36

13.242

17.743

$4.266 \quad 14.411$

$10.74 \quad 3.778$

$\begin{array}{ll}1.6 & 1.402\end{array}$

\begin{tabular}{lrrr} 
& \multicolumn{3}{c}{ MEAN } \\
Preop Day 1 & 73.24 & 4.99 & 12.96 \\
Amplitude & Acrophase \\
Preop Day 2 & 73.69 & 6.44 & 13.68 \\
In Hospltal Day 1 & 71.53 & 6.95 & 10.58 \\
In Hospltal Day 2 & 70.09 & 4.20 & 16.04 \\
Postop Day 1 & 74.39 & 5.02 & 8.86 \\
Postop Day 2 & 77.29 & 5.58 & 9.87
\end{tabular}

\begin{tabular}{rrr}
17.037 & 2.52 & 16.302 \\
9.553 & 17.122 & 16.875 \\
5.07 & 16.958 & 3.417 \\
16.912 & 4.477 & 24.815 \\
21.199 & 7.944 & 1.082 \\
9.693 & 17.8 & 24.72 \\
& & \\
& & \\
\multicolumn{4}{c}{ Mesor } & Amplitude & Acrophase \\
73.24 & 4.99 & 12.96 \\
73.69 & 6.44 & 13.68 \\
71.53 & 6.95 & 10.58 \\
70.09 & 4.20 & 16.04 \\
74.39 & 5.02 & 8.86 \\
77.29 & 5.58 & 9.87
\end{tabular}

Postop Day 2

(n)




\section{Pulse}

Mesor

Preop Day 1

Preop Day 2

In Hospltal Day 1

In Hospltal Day 2

Postop Day 1

Postop Day 2

Amplitude

Preop Day 1

Preop Day 2

In Hospltal Day 1

In Hospltal Day 2

Postop Day 1

U

Patlent 11 Patlent 12 Patlent 15 Patlent 18 Patlent 14 Patlent 17 Pallent 21 Patlent 19 Patlent 22 Patlent 23

$\begin{array}{ll}92.869 & 91.907 \\ 83.981 & 97.742 \\ 61.673 & 86.234 \\ 68.851 & 84.794 \\ 90.044 & 84.727\end{array}$

68.566

68.810

82.065

$64.776 \quad 76.669$

$61.549 \quad 82.375$

81.99876 .538

$74.407 \quad 72.544$

84.999

78.532

77.744

82.568

89.960

70.795

$74.694 \quad 70.858$

$71.814 \quad 86.297$

84.379

99.564

94.168

73.561

63.134

102.202

80.443

89.216

80.179

87.404

83.383

84.685

81.429

93.334

96.194

91.226

99.935

87.214

$\begin{array}{rr}10.202 & 5.624 \\ 2.191 & 9.307 \\ 8.990 & 11.637 \\ 9.571 & 6.610 \\ 4.880 & 3.617 \\ 1.837 & 3.356\end{array}$

7.145
6.131
6.606
5.238
10.350
4.283

$\begin{array}{rr}6.094 & 2.274 \\ 1.317 & 22.125 \\ 3.235 & 7.350 \\ 5.690 & 0.767 \\ 10.857 & 7.806 \\ 4.631 & 3.785\end{array}$

5.877

\subsection{5}

2.154

4.450

6.990

2.529

$\begin{array}{rr}6.222 & 5.335 \\ 3.025 & 11.043 \\ 12.431 & 8.329 \\ 15.820 & 12.383 \\ 3.835 & 3.590 \\ 16.055 & 11.332\end{array}$

1.523

9.726

8.600

12.053

5.160

9.511

15.104

15.798

4.835

14.514

13.502

4.454

Acrophase

Preop Day 1

Preop Day 2

In Hospltal Day 1

In Hospltal Day 2

Postop Day 1

Postop Day 2

$\begin{array}{crr}22.247 & 2.170 & 10.555 \\ 18.026 & 2.784 & 11.508 \\ 22.914 & 23.632 & 1.907 \\ 20.739 & 16.414 & 19.756 \\ 23.051 & 18.014 & 1.557 \\ 22.200 & 3.356 & 2.405 \\ & & \\ & & \\ & \text { MEAN } & \\ \text { Mesor } & \text { Amplltude Acrophase } \\ 80.643 & 6.540 & 13.987 \\ 83.792 & 8.306 & 11.433 \\ 75.939 & 7.417 & 12.500 \\ 77.969 & 8.710 & 13.097 \\ 82.832 & 7.059 & 13.065 \\ 84.425 & 6.177 & 9.072\end{array}$

2.4


Temperature

Mesor

Preop Day 1

Preop Day 2

In Hospltal Day 1

In Hospltal Day 2

Postop Day 1

Postop Day 2

Amplitude

Preop Day 1

Preop Day 2

In Hospltal Day 1

In Hospttal Day 2

Postop Day 1

Postop Day 2

$\omega$

Acrophase

Preop Day 1

Preop Day 2

In Hospltal Day 1

In Hospltal Day 2

Postop Day 1

Postop Day 2

Preop Day 1

Preop Day 2

In Hospttal Day 1

In Hospltal Day 2

Postop Day 1

Postop Day 2
Patlent 11 Patlent 12 Patlent 15 Patlent 18 Patlent 14 Patlent 17 Patlent 21 Patlent 19 Patlent 22 Patlent 23

$\begin{array}{ll}97.254 & 99.136 \\ 98.751 & 98.509 \\ 97.910 & 98.772 \\ 98.044 & 98.415 \\ 97.989 & 98.412 \\ 97.360 & 97.807\end{array}$

$\begin{array}{rr}97.555 & 97.704 \\ 98.273 & 97.221 \\ 97.748 & 97.984 \\ 98.299 & 99.615 \\ 101.523 & 98.321 \\ 98.976 & 98.376\end{array}$

98.229

97.743

97.726

98.051

$98.243 \quad 98.134$

$97.526 \quad 97.569$

$97.161 \quad 97.837$

$96.935 \quad 98.268$

$\mathbf{9 7 . 5 5 8}$
$\mathbf{9 7 . 8 5 7}$
$\mathbf{9 5 . 7 4 6}$
96.137
96.865
$\mathbf{9 8 . 2 6 1}$

96.639

97.904

$96.270 \quad 98.398$

$96.554 \quad 98.757$

$97.110 \quad 98.856$

$97.513 \quad 98.737$

96.61298 .848

97.944

97.897

98.643

99.297

98.478

$\begin{array}{lll}0.677 & 0.114 & 0.681\end{array}$

1.159

1346

0.886

1.511

1.854

1.672

0.749

0.812

0.531

0.792

0.798

0.754

3.707

0.513

0.580

0.514

0.520

0.279

0.506

0.148

0.715
1.154
0.199
0.311
0.153
0.290

1.137

1.170

0.159

0.468

0.199

0.237

0.793
0.434
1.460
1.784
0.765
0.246

0.158

1.190

0.772

0.881

0.360

0.180

0.819

0.165

0.490

0.276

0.206

0.302
1.448

0.544

2.700

0.929

0.284

$\begin{array}{rrr}21.050 & 19.412 & 13.764 \\ 2.720 & 14.139 & 19.657 \\ 2.451 & 22.620 & 16.388 \\ 21.556 & 22.135 & 15.491 \\ 19.141 & 22.798 & 4.199 \\ 19.515 & 11.828 & 3.860 \\ & & \\ & & \\ \text { Mesor } & \text { Amplitude Acrophase } \\ 97.765 & 0.712 & 12.506 \\ 97.897 & 0.882 & 13.778 \\ 97.775 & 0.777 & 11.640 \\ 98.021 & 0.955 & 14.402 \\ 98.366 & 0.852 & 14.533 \\ 97.992 & 0.424 & 15.893\end{array}$

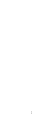

23.587
24.074
16.714
14.059
17.110
15.686

2.028

14.377

20.604

1.174

12.617

15.030
2.661
6.043
1.794
21.904
24.077
18.786

18.959

2.156

16.552

5.038

19.450
Mesor Amplitude Acrophase 
MEAN URINE CORTISOL LEVEL

$\begin{array}{lr}\text { PATIENT \#11 } & \text { Mean } \\ \text { Preop Day \#1 } & 14.6 \\ \text { Preop Day \#2 } & 13.8 \\ \text { Inhospital \#1 } & 4.4 \\ \text { Inhospital \#2 } & 7.5 \\ \text { Postop Day \#1 } & 11.1 \\ \text { Postop Day \#2 } & 11.2\end{array}$

Patient \#12 Mean

Preop Day \#1 15.8

Preop Day \#2 21.2

Inhospital \#1 11.7

Postop Day \#1 9.5

Patient \#14 Mean

Preop Day \#1 17.4

Preop Day \#2 17.9

Inhospital \#1 37.3

Inhospital \#2 19.7

Postop Day \#1 16.1

Postop Day \#2 21.4

Patient \#15 Meān

Preop Day \#1 8.2

Preop Day \#2 7.1

Inhospital \#1 5.0

Inhospital \#2 3.5

Postop Day \#1 2.8

Patient \#17 Mean

Preop Day \#1 4.5

Preop Day \#2 4.3

Inhospital \#1 7.2

Inhospital \#2 2.1

Postop Day \#1 0.9

Postop Day \#2 2.0 
Patient \#18 Mean

Preop Day \#1 12.6

Preop Day \#2 13.5

Inhospital \#1 4.9

Inhospital \#2 18.9

Postop Day \#1 6.6

Postop Day \#2 3.6

Patient \#19 Mean

Preop Day \#1 4.4

Inhospital \#1 4.1

Inhospital \#2 1.6*

Postop Day \#1 0.6 *

Postop Day \#2 $0.8 *$

$\star$ Discarded Specimens

Patient \#21 Mean

$\begin{array}{lr}\text { Preop Day \#1 } & 13.1 \\ \text { Preop Day \#2 } & 5.3 \\ \text { Inhospital \#1 } & 3.9 \\ \text { Inhospital \#2 } & 2.4 \\ \text { Postop Day \#1 } & 6.0 \\ \text { Postop Day \#2 } & 3.0\end{array}$

Patient \#22 Mean

Preop Day \#1 16.1

Preop Day \#2 14.6

Inhospital \#1 14.6

Inhospital \#2 11.6

Postop Day \#1 10.3

Postop Day \#2 6.0

Patient \#23 Mean

Preop Day \#1 22.1

Preop Day \#2 *

Inhospital \#1 $50+$

Inhospital \#2 37.2

Postop Day \#1 8.9

Postop Day \#2 20.1

*Discarded Specimens 


\section{URINE CORTISOL LEVELS}

Patient \#11

Preoperative Levels

$080012.0 \quad 080022.0$

$\begin{array}{llll}1600 & 17.2 & 1600 & 5.5\end{array}$

Inhospital Levels

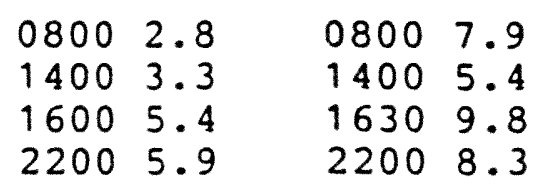

Postoperative Levels

$\begin{array}{llll}\# 0800 & 15.2 & 0800 & 11.3\end{array}$

$1600 \quad 6.9 \quad 1600 \quad 11.4$

Patient \#12

Preoperative Levels

$\begin{array}{llll}0800 & 22.2 & 0800 & 22.1\end{array}$

$\begin{array}{llll}1600 & 9.3 & 1600 \quad 20.2\end{array}$

Inhospital Levels

160011.7 *

Postoperative Levels

08008.6

160010.3

Patient \#14

Preoperative Levels

$0800 \quad 17.4 \quad 0800 \quad 18.3$

$1600 \quad 17.6$ 
Inhospital Levels

$\begin{array}{llc}0800 & 37.3 & 0800 \quad 21.3 \\ 1600 & 18.1 & *\end{array}$

Postoperative Levels

$\begin{array}{cccc}0800 & 16.1 & 0800 & 30.7 \\ 1600 & 12.0 & & \star\end{array}$

Patient \#15

Preoperative Levels

$08007.20800 \quad 6.5$

$\begin{array}{lllll}1600 & 9.1 & 1600 & 7.7\end{array}$

Inhospital Levels

$\begin{array}{llll}1400 & 6.5 & 0700 & 2.6\end{array}$

$2300 \quad 3.5 \quad 1600 \quad 1.8$

Postoperative Levels

08003.9

16001.6

$\star$

Patient \#17

Preoperative Levels

08007.208007 .0

$\begin{array}{llll}1600 & 1.8 & 1600 & 1.6\end{array}$

Inhospital Levels

$\begin{array}{llll}0800 & 8.4 & 0800 & 3.7\end{array}$

$\begin{array}{llll}1600 & 6.0 & 1600 \quad 0.5\end{array}$

Postoperative Levels

$0800 \quad 0.9 \quad 0800 \quad 3.0$

$\begin{array}{llll}1600 & 0.9 & 1600 & 0.9\end{array}$ 
Patient $\# 18$

Preoperative Levels

$080017.5 \quad 0800 \quad 18.3$

$\begin{array}{llll}1600 & 7.7 & 1600 & 8.7\end{array}$

Inhospital Levels

$\begin{array}{llll}0800 & 5.3 & 0800 & 30.1\end{array}$

$\begin{array}{llll}1600 & 4.4 & 1600 \quad 7.6\end{array}$

Postoperative Levels

$\begin{array}{llll}0800 & 7.2 & 0800 & 2.6\end{array}$

$16005.9 \quad 1600 \quad 6.6$

Patient \#19

Preoperative Levels
$08006.7 \quad *$
16002.0

Inhospital Levels

08006.2 *

$16001.9 \quad 1600 \quad 1.6$

Postoperative Levels

$\begin{array}{cccc}0800 & 0.6 & 0800 & 0.8\end{array}$

Patient \#21

Preoperative Levels

$\begin{array}{llll}0800 & 23.9 & 0800 & 6.2\end{array}$

$\begin{array}{llll}1600 & 2.3 & 1600 & 4.4\end{array}$

Inhospital Levels

$\begin{array}{lllll}0800 & 3.8 & 0800 & 1.6\end{array}$

$\begin{array}{lllll}1600 & 3.9 & 1600 & 3.2\end{array}$ 


$$
\begin{aligned}
& \text { Postoperative Levels } \\
& \begin{array}{llll}
0800 & 10.7 & 0800 & 5.3
\end{array} \\
& 16001.2 \quad 1600 \quad 0.6
\end{aligned}
$$

Patient \#22

$$
\begin{aligned}
& \text { Preoperative Levels } \\
& 080016.1 \quad 08009.5 \\
& \star
\end{aligned}
$$

Inhospital Levels

$$
\begin{array}{lll}
0800 & 14.6 & 080021.5 \\
* & & 16001.6
\end{array}
$$

Postoperative Levels

$$
\begin{array}{llll}
0800 & 15.0 & 0800 & 8.9 \\
1600 & 5.5 & 1600 & 3.0
\end{array}
$$

Patient \#23

Preoperative Levels * 080017.1

$\begin{array}{llll}1600 & 21.0 & 1600 \quad 27.1\end{array}$

Inhospital Levels

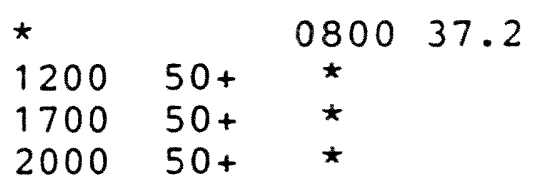

Postoperative Levels

080012.0 *

$16005.8 \quad 20.1$

* Missed or Discarded Specimens 
FLORIDA INTERNATIONAL UNIVERSITY

UNIVERSITY RESEARCH COUNCIL (URC)

APPLICATION FOR APPROVAL OF RESEARCH INVOLVING HUMAN SUBJECTS

PRINCIPAL DNVESTIGATOR: Jeanne Anne Abdou S5: $098-42-9754$ Address: 313 Cape Coral Parkway, \#4; Cape Cora1 FL Phone ": $941-542-5793$ Position: | | Feculty |X| Graduate Student | | Undergraduate Student 1 | Other (Specify)

3. FACUITY SUPERVISOR (if $P I$ is a student): Dr. Luz Porter

4. STATUS OF PROTECT REVIEW:

IXINew project I / Revision of previously approved project I I Continuation of approved project BRIEF DESCRIPTION OF SUBJECTS

Number of subjecis: 10

Check all of the following calegones that describe your research subjects:

$|\mathrm{X}|$ Males

(x) Females

1 ) Minors (under 18 years old)

1 I Siudents (Please Specily):

1 I Persons With Physical Disabilities (Please Specify):

1 I Persons With MentalPsychological Disabilities (Please Specify):

1 I Persons With Physical or Mencal Healch Problems (Please Specify):

1 | Persons With No Known Disabilities and No Known Health Probiems

1 I Fnsoners

1 I Pregrant women, feruses, feul material or placenus (Plesse Specify):

1 I Persons in Some Type of Properm (Please Specify):

1 I Other Peninent Information (Please Spocify):

6. TYPE OF REVIEW REQUESTED (Soe pies 6 \& of the Information for Experimenters booklet):

1 I Exempi: Cwetory $(s)$ :

[X] Expedilod Review: Caesory $m(3): 2,3$

1 I Full URC Review (Cm be neuthe Exemprod nox Expediled) 
.APPLCATION FOR APPROVAL OF RESEARCH INVOLVING HUMAN SUBJECTS

\section{RESE.ARCH OBJECTIVES}

The main objective of this research is to determine the effects of circadian rhythm disruption in post-surgical patients and their post-surgical recovery. Specifically, this study will focus on achieving the following objectives:

1.) Identify the effects of anesthesia/surgery on the circadian rhythm in postsurgical patients.

2.) Determine the mean time interval needed for resynchrony of disrupted circadian thỵthm in postsurgical patients.

3.) Determine the relationship between circadian thythm disruption in different types of surgical procedures.

\section{SUBJECT RECRUTTMENT}

The sample in this study are comprised of five males and five females, eighteen years and older who will serve as their own presurgical control. Due to the nature and complexity of data retrieval a sample size of ten is sufficient.

The target population for this study consists of patients hospitalized for surgery. The sample will be derived from surgical patients admitted in an urban hospital in southwest Florida. Sampling will be done by convenience utilizing a computerized list of patients scheduled for preoperative testing. This will include patients from telemetry and non-telemetry units, neurology and oncology units. orthopedic and general vascular surgery units, and critical care surgical units. 
The prospective subjects are informed about the purpose and procedure of the study. The risks and benefits are explained and they are told that they can withdraw at any time. Those who agree to participate are requested to sign an informed consent and are assured of the confidentiality of the data collected.

\section{BENEFITS}

Subjects will receive no direct benefit from this study. However, the results of this research will be made available to them upon their request. The anticipated benefits to society is the potential improvement of an individual's post-surgical period. Findings from this study will assist health professionals in providing individualized patient care.

\section{INFORMED CONSENT}

The introductory letter includes information regarding the patients right to choose whether or not to participate in this study. The consent form further explains that their decision to decline to participate will not affect the services they receive from their health care providers or affect their hospitalization. The participants will be assured that all the information will be kept confidential and anonymous. An individual's voluntary permission to participate in this research is exemplified by a signed and witnessed consent form.

\section{CONFIDENTIALIT: OF DATA}

Participants' information will be kept anonymous. as no names or identifying markings will be utilized. The raw data are accessible only to the principal investigator. All data will be kept in a 
secured, locked file cabinet for a minimum period of three years, at which time all information will be destroyed appropriately.

\section{METHODS AND PROCEDURES}

The comparative correlational research design will be used in this study to test the hypotheses. To determine circadian rhythm disruption, body temperature, heart rate, and blood pressure measurements will be recorded. Post-surgical recovery will be measured by locomotor activity utilizing patients' activity diary and urine cortisol level. Data will be collected from the sample of ten subjects during the pre-operative and post-operative periods: while hospitalized and then 2 days after hospital discharge. Potential subjects will be approached in person, on the day of their pre-operative testing appointment.

In order to evaluate a circadian thythm disturbance, the following criteria will be observed:

A.) Body temperature, heart rate and blood pressure measurements every 2 hours during the hours of $0800-2200$ for 48 hours. Specifically, 2 days before surgery, post-operative day $\# 1$ and $\# 2$, and 2 consecutive days after hospital discharge.

B.) Activity level data, as described in patient activity diaries and urine cortisol levels obtained four times a day.

Data collection will start once approval from appropriate review committees (Florida International University URC. and agency IRB) is secured. The data will be collected seven times a day at two hour intervals during normal waking hours, between the hours of 0800 and 2200 , 
during the two pre-operative days, the first and second days post-operatively, and two consecutive days after hospital discharge. The data collected at these specific times are temperature, apical heart rate. systolic blood pressure. and diastolic blood pressure. Urine samples will also be collected and frozen four times a day for subsequent assay for cortisol. Post-operative urine samples will be collected from an indwelling urinary drainage system. Pre and post hospitalization voided urine samples will be collected upon awakening and berween the hours of 2:00pm and 4:00pm. Each subject will receive eight clean specimen containers. During data collection the subjects will be asked to complete a brief activity diary to correlate with urine cortisol levels. Sampling times were chosen to approximate a typical wake/sleep cycle and minimize the effects of data collection.

Blood pressure measurements are obtained by Datascope automatic blood pressure cuffs every two hours and recorded in the internal memory. The Datascope is used to standardize procedure and prevent errors in interrater reliability.

Body temperature measurements are obtained by standard probe tympanic electronic thermometers. The thermometers are equipped with liquid crystal display (LCD) and checked for accuracy everyday using the proper methods recommended by the manufacturer. To ensure uniformity, temperatures are taken tympanically every two hours and recorded on a graph sheet. Tympanic temperatures. apical heart rate and brachial blood pressures are monitored according to standardized protocols. 
Activity level will be documented by participant written activity diaries and correlated to urine corisol levels. The urine specimens will be collected and frozen for subsequent assay. The urine specimens will be assayed for $17-$ ketosteroids using modified colormetric assay.

The data will be recorded on individualized graph sheets for each subject. The graph sheets will be placed in a notebook secured in a locked fle cabinet for the duration of the study.

\section{STMMULUS MATERIAL}

1.) Datascope automatic blood pressure cuff as described in item $\# 12$ on page 4 .

2.) Tympanic electronic thermometer as described in item $\# 12$ on page 4 .

3.) Subject recruitment letter.

4.) Consent form for participants.

5.) Consent to conduct research at Cape Coral Hospital.

6.) Acceptance letter from the Vice President of Patient Services at Cape Coral Hospital.

\section{RISKS TO SUBJECTS}

The purpose of this study is to explore circadian rhythm disnuption in post-surgical recovery. No physical, psychological social, economic or legal risk should arise as a result of this study. The participants of this study will receive health care according to the hospitals standard policies and procedures. Those patients who choose not to participate are assured of the same delivery of health care. The participants of the study do so voluntarily. All information obtained is 
collected anomymousty and kept confidential. If any questions or concerns arise, the participants have access to myself, as principal investigator. The hospital administration is fully aware of the study and are available to offer assistance to any participant. The participants may also address any concerns to the physician providing medical care, or to the faculty supervisor for any questions regarding research methodology. 


\section{AFFIRMATION OF COMPLIANCE AND ACCEPTANCE OF RESPONSIBILTI}

1 aesee 10 follow the procedures outlined in this summary dexcription and any atuchments. 1 undersand that no coniact may be initiated with subjects until I have received approval of these procedures from the URC and have complied with any modifications required in conncetion with that approval. I undersund inat additions to or changes in the procedures involving human subjects can conly be made after approval of the URC. I undersiand that I must promplly report to the URC any problems with the righis or welfere of the human subjects. I undersiand and will follow Florida International University's policies conceming research with human subjects I will do evergithing in my powet 10 procect the righis and welfare of human subjects in my research project.

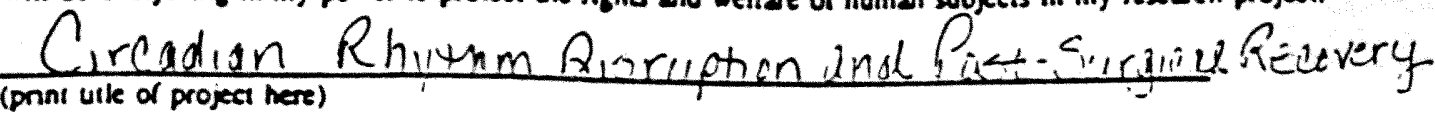

Srenziure of Principal Invessigator

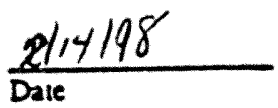

Jeanne Anne Abolou

Prnied name of Principal Investigalox

If the $\mathrm{Pl}$ is a student. the faculty supervisor must sign below.

I have read this application and assume responsibility for is eceuracy and for supervision of the proposed resench proxet.

Signatuff of Faculty Supervisor

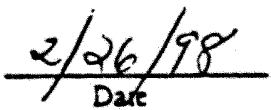

Luz S. PRTER

Prnied name of Faculty Supervisor

ACTION RECOMOMENDE BY URC * For URC use only"•

Date: $3 / 5 / 98$ I 4 Approved I I Changesclarifications Requested I I Require Full Board Review Signature of URC Chaiperion tor BERNARD GERSTMAN

SUMAN KAKAR FOR BERNARD GERSTMAN

Prinued name of URC Chaisperson

Date:

I Approved I J Changesclinificaions Requested

I I Require Full Board Review

Signavure of URC Cheiperson

Dax: 
March 5. 1998

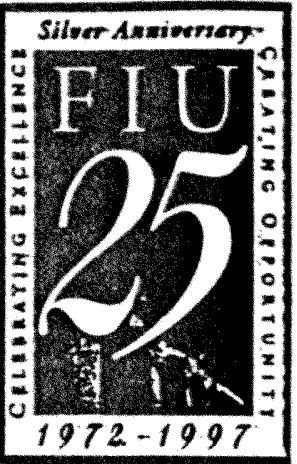

Ms. Jeane Anne Abdou

(Dr. Luz Porter)

AC 203

Florida International University

North Miami Campus

North Miami Fl 33181

Dear Ms. Abdou:

I am pleased to inform you that your research proposal titled "Circadian Rhythm Disruption and Post-Surgical Recovery" has been reviewed and approved. Please note that you are required to conduct your research and all related procedures in the exact same manner as indicated in the proposal without any exception. I wish you all the best.

Sincerely

Suman Kakar. Ph.D.

cc: Professor Gerstman

URC Chairperson

School of Policy and Management - College of Urban and Public Affairs Academic One, Suire 267, North Campus, North Miami, Florida 33181 


\section{Dear Client,}

My name is Jeanne Abdou. I am a graduate nursing student at Florida International University of Miami, Florida. As a requirement of a Masters degree in Nursing, 1 will be conducting a research project to examine the physiological effects of surgery and anesthesia on circadian rhythm. Circadian rhythm is defined as an individuals biological pattern which is repeated every 24 hours, and evidenced by changes in temperature, blood pressure, pulse, and components seen in urine tests.

Circadian rhythm help maintain man's optimal health. Surgery and anesthesia temporarily may alter the biological patterns listed above. Nurses assist patients in post-surgical recovery and restore pre-surgical health. Research studies involving the effects of surgery and anesthesia on circadian rhythm provide useful information. Nurses will obtain further knowledge in caring for patients following surgery.

You have been selected to participate in this study because you are going to have surgery and receive general anesthesia. Approximately 10 patients will be involved in this study. If you are agreeable to participate in the study, please read and sign the attached consent form.

There are no risks to you as a participant of this study. You will receive no direct benefit from participating in this study. Your health care and hospitalization will not be affected whether or not you choose to participate in this study. The results of this study will be made available to you upon your request. All information obtained will be kept confidential. Participants will remain anonymous, as no names or identifying markings will be reported. If you choose not to participate, the services you receive from your health care provider will not be affected: all data collected will be secured until the completion of this study, at which time all information will be destroyed. The information obtained will be accessible to myself, the researcher, and the research committee members.

Your participation in this study is greatly appreciated. I am available to answer any questions or concerns that may arise. The hospital administration is aware of this research study and are available to address any concerns. My research committee chairperson, Dr. Luz Poner, may also be reached at (305) 919-5845.

Thank you for your cooperation.

Sincerely,

Jeanne Anne Abdou, RN, BSN

Jeanne Anne Abdou

313 Cape Coral Pkwy, \#4

Cape Coral, Florida 33904

H. (941) 542-5793

W.(941) 574-0375 


\section{Informed Consent \\ Circadian Rhythm Disruption and Post Surgical Recovery}

I freety and voluntarity consent to be a participant in the research project entitled Circadian Rhythm Disruption and Post Sungical Recovery to be conducted at Florida International University during the spring semester, 1998, with Jeanne Abdou as principal investigator. I have been told that this experiment will last 6 days. Specifically, 2 days pre surgery, postoperative days $\# 1$ and $\# 2$ and 2 consecutive days after hospital discharge.

I understand that the purpose of this research is to investigate the disturbance of circadian rhythms in post surgical patients and to determine its effect in the post surgical recovery period.

I understand that the research procedure will be as follows: Temperature, pulse, and blood pressure measurements will be taken every 2 hours during the hours of 8:00am and 10:00pm. The pulse and blood pressure measurements will be taken by an automatic blood pressure machine. Body temperatures will be taken with a tympanic thermometer (probe in the ear). The days involved in the procedure are 2 days prior to surgery, 2 days after surgery, and 2 davs after hospital discharge. Urine samples will be collected 4 times a day during hospitalization, and 2 times a day during the pre surgical and post hospital period. Urine samples will be collected upon awakening and between the hours of $2: 00 \mathrm{pm}$ and $4: 00 \mathrm{pm}$. I will be completing a brief diary describing my activity throughout the day, ie. eating, walking, bathing, watching television, etc.

I understand that there are no known risks or benefits involved in my participation in this study. I have been told that all my information will be kept anonymous and confidential.

I understand that I may withdraw my consent and discontinue my participation in this research project at any time with no negative consequences. I have been given the right to ask questions conceming the procedure, and any questions have been answered to my satisfaction.

I understand that if I desire information about this research I should contact Jeanne Abdou. principal investigator, at (941) 542-5793 or Dr. Luz Porter, faculty supervisor, at (305) 919-5845.

I have read and understand the above.

Participant's signature

Date

I have explained and detined in detail the research procedure in which the participant has agreed to participate. and have offered himher a copy of this informed consent form.

Principal investigator's signature

Date 
January 14,1998

\author{
Ms. Donna Giannuzzi \\ Vice President of Patient Services \\ Cape Coral Hospital \\ 636 Del Prado Boulevard \\ Cape Coral, FL 33990
}

\title{
Dear Donna,
}

I am currently enrolled at Florida International University Graduate Nursing Program. I am conducting a Masters Thesis on Circadian Rhythm and Post-Surgical Recovery.

I am requesting permission to conduct my research study at Cape Coral Hospital. The research study requires monitoring the blood pressure, pulse, temperature and urine assay for catacholimes on ten surgical patients. This study will not generate any expense for the participants. My research is committed to the highest degree of privacy and confidentiality.

Thank you in advance for your consideration of this study. I look forward to working with you and your nursing staff during my research study. If you have any questions, feel free to contact me. My phone number is 542-5793.

Sincerely,

Jeanne Abdou, R.N. 


\section{CAPE CORAL HOSPITAL}

January 16, 1998

TO: Jeanne Abdou, R.N.

FROM: Donna Giannuzzi, R.N. D2

Vice President of Patient Services

SUBJECT: Graduate Research

I am pleased you have chosen Cape Coral Hospital to conduct your graduate research. As you know, it is critical when accessing patient information that confidentiality of patient records be maintained at all times. I am confident that you will respect this.

We look forward to working with you. If I can be of further assistance, please let me know.

$D G / s m$

cc: Jan Offret, R.N.

Director, Critical Care Services and Emergency Department 